\title{
ALGEBRAIC ENTROPY OF SHIFT ENDOMORPHISMS ON ABELIAN GROUPS
}

\author{
MARYAM AKHAVIN, FATEMAH AYATOLLAH ZADEH SHIRAZI, DIKRAN DIKRANJAN, \\ ANNA GIORDANO BRUNO, AND AREZOO HOSSEINI
}

Dedicated to Prof. A. Chademan, with best wishes for him

\begin{abstract}
For every finite-to-one map $\lambda: \Gamma \rightarrow \Gamma$ and for every abelian group $K$, the generalized shift $\sigma_{\lambda}$ of the direct sum $\bigoplus_{\Gamma} K$ is the endomorphism defined by $\left(x_{i}\right)_{i \in \Gamma} \mapsto\left(x_{\lambda(i)}\right)_{i \in \Gamma}$ 3]. In this paper we analyze and compute the algebraic entropy of a generalized shift, which turns out to depend on the cardinality of $K$, but mainly on the function $\lambda$. We give many examples showing that the generalized shifts provide a very useful universal tool for producing counter-examples.
\end{abstract}

We denote by $\mathbb{Z}, \mathbb{P}$, and $\mathbb{N}$ respectively the set of integers, the set of primes, and the set of natural numbers; moreover $\mathbb{N}_{0}=\mathbb{N} \cup\{0\}$. For a set $\Gamma, \mathcal{P}_{\text {fin }}(\Gamma)$ denotes the family of all finite subsets of $\Gamma$. For a set $\Lambda$ and an abelian group $G$ we denote by $G^{\Lambda}$ the direct product $\prod_{i \in \Lambda} G_{i}$, and by $G^{(\Lambda)}$ the direct sum $\bigoplus_{i \in \Lambda} G_{i}$, where all $G_{i}=G$. For a set $X, n \in \mathbb{N}$, and a function $f: X \rightarrow X$ let $\operatorname{Per}(f)$ be the set of all periodic points and $\operatorname{Per}_{n}(f)$ the set of all periodic points of order at most $n$ of $f$ in $X$.

\section{INTRODUCTION}

The measure entropy was introduced by Kolmogorov and Sinai in ergodic theory in the mid fifties of the last century. Some ten years later Adler, Konheim, and McAndrew [1] introduced the notion of topological entropy $h_{t o p}(T)$ of a continuous self-map $T: X \rightarrow X$ of a compact topological space $X$. A prominent example in both cases is provided by the Bernoulli shifts. Since these shifts are the core of this paper, we introduce them here in full detail.

Example 1.1. Let $K$ be a non-trivial finite group with neutral element $e_{K}$.

(a) The two-sided Bernoulli shift $\bar{\beta}_{K}$ of the group $K^{\mathbb{Z}}$ is defined by $\bar{\beta}_{K}\left(\ldots, x_{0}, x_{1}, x_{2}, \ldots\right)=\left(\ldots, x_{-1}, x_{0}, x_{1}, \ldots\right)$, (i.e., $\bar{\beta}_{K}\left(\left(x_{n}\right)_{n \in \mathbb{Z}}\right)=\left(x_{n-1}\right)_{n \in \mathbb{Z}}$, for $\left.\left(x_{n}\right)_{n \in \mathbb{Z}} \in K^{\mathbb{Z}}\right)$.

(b) The right Bernoulli shift $\beta_{K}$ (respectively, left Bernoulli shift ${ }_{K} \beta$ ) of the group $K^{\mathbb{N}_{0}}$ is defined by

$\beta_{K}\left(x_{1}, x_{2}, x_{3}, \ldots\right)=\left(e_{K}, x_{1}, x_{2}, \ldots\right),\left(\right.$ respectively, ${ }_{K} \beta\left(x_{0}, x_{1}, x_{2}, \ldots\right)=\left(x_{1}, x_{2}, x_{3}, \ldots\right)$.

The standard product measure of the compact group $K^{\mathbb{Z}}$ (respectively, $K^{\mathbb{N}_{0}}$ ) coincides with its Haar measure and $\bar{\beta}_{K}$ (respectively, ${ }_{K} \beta$ ) is a measure-preserving continuous automorphism (respectively, endomorphism) with topological entropy $\log |K|$ coinciding with the measure entropy. This explains their relevance to both ergodic theory and topological dynamics.

The right Bernoulli shift $\beta_{K}$ of $K^{\mathbb{N}_{0}}$ is less relevant in this respect for two reasons: it is not measurepreserving (so not relevant for ergodic theory) and its topological entropy is 0 .

A possible definition of algebraic entropy for endomorphisms of abelian groups was briefly mentioned in [1. Later on, in 1975 in [6] Weiss defined the algebraic entropy as follows: let $G$ be an abelian group and $F$ be a finite subgroup of $G$; for an endomorphism $\phi: G \rightarrow G$ and $n \in \mathbb{N}$, let $T_{n}(\phi, F):=$ $F+\phi(F)+\ldots+\phi^{n-1}(F)$ be the $n$-trajectory of $F$ with respect to $\phi$. The algebraic entropy of $\phi$ with respect to $F$ is

and the algebraic entropy of $\phi: G \rightarrow G$ is

$$
H(\phi, F):=\lim _{n \rightarrow+\infty} \frac{\log \left|T_{n}(\phi, F)\right|}{n},
$$

$$
\operatorname{ent}(\phi)=\sup \{H(\phi, F): F \text { is a finite subgroup of } G\} .
$$

1991 Mathematics Subject Classification. 37A35, 20K01, 20K10, 20 K30.

Key words and phrases. algebraic entropy, abelian group, generalized shift, shift, trajectory. 
Since the definition is based on finite subgroups $F$, and in particular $F$ is contained in the torsion part $t(G)$ of $G$, the algebraic entropy depends only on the restriction of $\phi$ on $t(G)$, that is ent $(\phi)=$ $\operatorname{ent}\left(\phi \uparrow_{t(G)}\right)$. The basic properties of the algebraic entropy can be found in [4, 6]. The most relevant of them, known also as Addition Theorem, can be found in $\$ 2$ (Theorem 2.3), which collects all relevant properties of the algebraic entropy used in this paper.

As far as the algebraic entropy is concerned, the right Bernoulli shift restricted to the direct sum $K^{\left(\mathbb{N}_{0}\right)}$ turned out to be more relevant (while the restriction of the left Bernoulli shift ${ }_{K} \beta \uparrow_{K^{\left(\mathbb{N}_{0}\right)}}$ has algebraic entropy 0, see Example 4.10). More precisely, for a non-trivial finite abelian group $K$ the restriction $\beta_{K} \Upsilon_{K^{\left(\mathbb{N}_{0}\right)}}$ has entropy $\log |K|$ [4. Example 1.9] and one can show that every function $f$ defined on all endomorphisms of torsion abelian groups with values in the extended non-negative reals and satisfying $f\left(\beta_{\mathbb{Z}(p)}\left\lceil_{\mathbb{Z}(p)^{\left(\mathbb{N}_{0}\right)}}\right)=\log |p|\right.$, the Addition Theorem and a few other natural properties (namely, Lemmas 2.1, 2.2 and Remark 2.4 (b)) must necessarily coincide with the algebraic entropy ent(-) 4, Theorem 6.1].

With the aim of computing the entropy of other endomorphisms of abelian groups, in this paper we consider a modification of the generalized shifts, introduced in [3].

Definition 1.2. 3] For a non-empty set $\Gamma$, an arbitrary map $\lambda: \Gamma \rightarrow \Gamma$ and an abelian group $K$ the generalized shift $\sigma_{\lambda, K}: K^{\Gamma} \rightarrow K^{\Gamma}$ is defined by $\left(x_{i}\right)_{i \in \Gamma} \mapsto\left(x_{\lambda(i)}\right)_{i \in \Gamma}$ for every $\left(x_{i}\right)_{i \in \Gamma} \in K^{\Gamma}$.

When there is no possibility of confusion we write $\sigma_{\lambda}$ instead of $\sigma_{\lambda, K}$. In case $|K|>1$, the subgroup $K^{(\Gamma)}$ of $K^{\Gamma}$ is $\sigma_{\lambda, K}$-invariant if and only if $\lambda$ has finite fibers (see Lemma 4.3), and it is possible to consider the restriction $\sigma_{\lambda, K}^{\oplus}=\sigma_{\lambda, K} \uparrow_{K^{(\Gamma)}}$ of $\sigma_{\lambda, K}$ to $K^{(\Gamma)}$. Again, when there is no possibility of confusion we write $\sigma_{\lambda, K}^{\oplus}$ simply as $\sigma_{\lambda}^{\oplus}, \sigma_{\lambda, K}$ or just $\sigma_{\lambda}$.

There is a close relation between the Bernoulli shifts and the generalized shifts. For example, the left Bernoulli shift and the two-sided Bernoulli shift are generalized shifts (see Examples 4.10 and 4.15 (d) respectively), while the right Bernoulli shift $\beta_{K} \uparrow_{K^{\left(\mathbb{N}_{0}\right)}}$ cannot be obtained as a generalized shift $\sigma_{\lambda}^{\oplus}$ from any function $\lambda: \mathbb{N}_{0} \rightarrow \mathbb{N}_{0}$. Nevertheless, it can be "approximated" quite well by the generalized shift $\sigma_{\psi}^{\oplus}$ of $K^{\left(\mathbb{N}_{0}\right)}$ induced by the map $\psi: \mathbb{N}_{0} \rightarrow \mathbb{N}_{0}$ defined by $\psi(i)=i-1$ for every $i>0$ and $\psi(0)=0$. Indeed, both $\sigma_{\psi}^{\oplus}$ and $\beta_{K} \uparrow_{K^{\left(\mathbb{N}_{0}\right)}}$ leave invariant the finite-index subgroup $H=K^{(\mathbb{N})}$ and $\sigma_{\psi}^{\oplus} \uparrow_{H}=\beta_{K} \uparrow_{H}$ (in particular, they have the same entropy $\log |K|$ ).

In this paper we compute the entropy of an arbitrary generalized shift $\sigma_{\lambda}^{\oplus}: K^{(\Gamma)} \rightarrow K^{(\Gamma)}$. More precisely, we show that $\operatorname{ent}\left(\sigma_{\lambda}^{\oplus}\right)$, depends only on combinatorial properties of the map $\lambda$ and the cardinality of $K$. To prove this we analyze the structure of the map $\lambda$ and, more specifically, its (iterated) counter-images (since, in some sense, the iterations of $\lambda$ and the iterations of $\sigma_{\lambda}$ "go in opposite directions"). Roughly speaking we decompose the generalized shift $\sigma_{\lambda}^{\oplus}$ in "independent elementary shifts" (as the generalized shift $\sigma_{\psi}^{\oplus}$ considered above), which have the same algebraic entropy as the right Bernoulli shift $\beta_{K} \uparrow_{K^{\left(N_{0}\right)}}$, and the number $s_{\lambda}$ of these independent elementary shifts, multiplied by $\log |K|$, gives precisely $\operatorname{ent}\left(\sigma_{\lambda}^{\oplus}\right)$ (see Theorem 4.14).

Convention. From now on we assume that $\Gamma$ is a non-empty set, $\lambda: \Gamma \rightarrow \Gamma$ is an arbitrary map, and $K$ is a non-trivial finite abelian group. We denote by $G_{\Gamma}$ the group $K^{(\Gamma)}$ and for a subset $A$ of $\Gamma$, we identify $K^{(A)}$ with the subgroup $\left\{x \in G_{\Gamma}: \operatorname{supp}(x) \subseteq A\right\}$ of $G_{\Gamma}$, and we denote $K^{(A)}$ by $G_{A}$. In case $A=\emptyset$ we assume that $G_{\emptyset}=\{0\}$. We denote the generalized shift based on $G_{\Gamma}$ and $\lambda: \Gamma \rightarrow \Gamma$ simply by $\sigma_{\lambda}$, writing it in some cases $\sigma_{\lambda, K}$, when we need to specify the group.

\section{BACKGROUND ON ALGEBRAIC ENTROPY}

We start collecting basic results on algebraic entropy, mainly from [4, 6].

Lemma 2.1. [6, Proposition 1.2] Let $G, H$ be abelian groups and $\phi: G \rightarrow G, \eta: H \rightarrow H$ endomorphisms. If there exists an isomorphism $\xi: G \rightarrow H$ such that $\phi=\xi^{-1} \eta \xi$, then $\operatorname{ent}(\phi)=\operatorname{ent}(\eta)$.

Lemma 2.2. [6, Proposition 1.3] Let $G$ be an abelian group and $\phi: G \rightarrow G$ an endomorphism. Then $\operatorname{ent}\left(\phi^{k}\right)=k \operatorname{ent}(\phi)$ for every non-negative integer $k$. If $\phi$ is an automorphism, then $\operatorname{ent}\left(\phi^{k}\right)=|k| \operatorname{ent}(\phi)$ for every $k \in \mathbb{Z}$.

The following is one of the main results on algebraic entropy. 
Theorem 2.3 (Addition Theorem). 4, Theorem 3.1] Let $G$ be a torsion abelian group, $\phi: G \rightarrow G$ an endomorphism and $H$ a $\phi$-invariant subgroup of $G$. If $\bar{\phi}: G / H \rightarrow G / H$ is the endomorphism induced on the quotient by $\phi$, then $\operatorname{ent}(\phi)=\operatorname{ent}\left(\phi \uparrow_{H}\right)+\operatorname{ent}(\bar{\phi})$.

Remark 2.4. Let $G$ be an abelian group and $\phi: G \rightarrow G$ an endomorphism.

(a) A particular case of the above theorem was proved in [6, Proposition 1.4]: if $n \in \mathbb{N}, G=\bigoplus_{i=1}^{n} G_{i}$ and $G_{i}$ is a $\phi$-invariant subgroup of $G$ for $i=1, \ldots, n$, then $\operatorname{ent}(\phi)=\sum_{i=1}^{n} \operatorname{ent}\left(\phi \uparrow_{G_{i}}\right)$.

(b) If the group $G$ is a direct limit of $\phi$-invariant subgroups $\left\{G_{i}: i \in I\right\}$, then $\operatorname{ent}(\phi)=\sup _{i \in I} \operatorname{ent}\left(\left.\phi\right|_{G_{i}}\right)$ 4. Proposition 1.6].

(c) Using (b), one can extend (a) to arbitrary direct sums $G=\bigoplus_{i \in I} G_{i}$.

Lemma 2.5. 4] Let $G$ be an abelian group and $\phi: G \rightarrow G$ an endomorphism.

(a) If $X$ is a set of generators of $G$ and for every $x \in X$ there exists $d_{x} \in \mathbb{N}$ such that $\phi^{d_{x}}(x)=0$, then $\operatorname{ent}(\phi)=0$.

(b) If $\phi$ is periodic, then $\operatorname{ent}(\phi)=0$.

\section{Strings AND AN EFFECTIVE EQUivalenCE RELATion}

Now we introduce a notion that will play a prominent role in the computation of the algebraic entropy of the generalized shifts.

Definition 3.1. (a) A string of $\lambda$ (in $\Gamma$ ) is an infinite sequence of pairwise distinct elements $S=$ $\left\{m_{t}\right\}_{-t \in \mathbb{N}_{0}}$ such that $\lambda\left(m_{t}\right)=m_{t+1}$ for every $-t \in \mathbb{N}$.

(b) Let $s_{\lambda}:=\sup \{|\mathcal{F}|: \mathcal{F}$ is a family of pairwise disjoint strings of $\lambda\}$, and

(c) $\Gamma^{+}:=\bigcap_{n=1}^{\infty} \lambda^{n}(\Gamma)$.

A string $S=\left\{m_{t}\right\}_{-t \in \mathbb{N}_{0}}$ of $\lambda$ in $\Gamma$ is said to be acyclic if $\lambda^{n}\left(m_{0}\right) \notin S$ for every $n \in \mathbb{N}$. The next claim is easy to prove.

Claim 3.2. Each string $S$ of $\lambda$ in $\Gamma$ contains an acyclic string $S^{\prime}$ of $\lambda$.

The importance of $\Gamma^{+}$consists in the fact that it contains all strings of $\lambda$ as well as all periodic points of $\lambda$. Obviously, $\Gamma^{+}=\Gamma$ if and only if $\lambda$ is surjective. In general, the restriction $\lambda \Gamma_{\Gamma^{+}}: \Gamma^{+} \rightarrow \Gamma^{+}$need not be surjective (but this holds true if $\lambda$ has finite fibers).

Consider the following equivalence relation: $i \Re_{\lambda} j$ in $\Gamma$ if and only if there exist $m, n \in \mathbb{N}_{0}$ such that $\lambda^{n}(i)=\lambda^{m}(j)$. Let $\alpha_{\lambda}:=\mid\left\{i / \Re_{\lambda} \in \Gamma / \Re_{\lambda}: i / \Re_{\lambda}\right.$ contains at least one string of $\left.\lambda\right\} \mid$. Obviously, $\alpha_{\lambda} \leq s_{\lambda}$.

Example 3.3. Suppose that $\lambda$ is injective.

(a) The relation $\Re_{\lambda}$ in this particular case becomes: $\Re_{\lambda}=\left\{(i, j) \in \Gamma \times \Gamma: \exists m \in \mathbb{Z}: i=\lambda^{m}(j)\right\}$.

(b) The relation $\Re_{\lambda}$ has three types of equivalence classes:

$\left(b_{1}\right)$ finite equivalence classes,

$\left(b_{2}\right)$ infinite equivalence classes contained in $\Gamma^{+}$(i.e., containing a string of $\lambda$ ), of the form

$$
\left\{\ldots, \lambda^{-1}(i), i, \lambda(i), \lambda^{2}(i), \ldots\right\}
$$

$\left(b_{3}\right)$ infinite equivalence classes non-contained in $\Gamma^{+}$(i.e., non-containing a string of $\lambda$ ), of the form $\left\{i, \lambda(i), \lambda^{2}(i), \ldots\right\}$ with $i \in \Gamma \backslash \lambda(\Gamma)$.

(c) Then $\alpha_{\lambda}$ is the number of the infinite equivalence classes in ( $\left.\mathrm{b}_{2}\right)$. Consequently $\alpha_{\lambda}=s_{\lambda}$.

Example 3.4. Let $\Gamma=\mathbb{N}_{0}$. For every $n \in \mathbb{N}$ let $\varphi_{n}: \Gamma \rightarrow \Gamma$ be defined by

$$
\varphi_{n}(m)= \begin{cases}0 & \text { if } m=0,1, \ldots, n, \\ m-1 & \text { otherwise }\end{cases}
$$


The diagram for $\varphi_{n}$ with $n>1$ is the following:

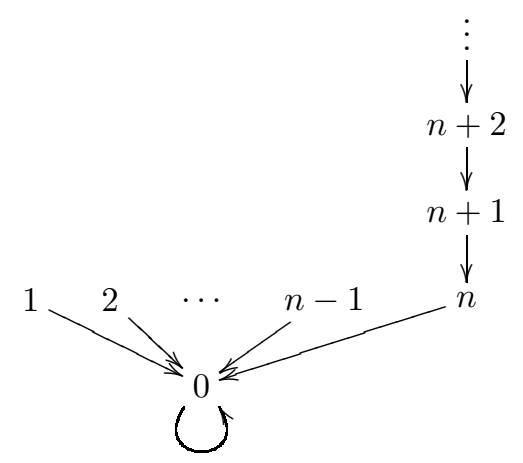

In this case $s_{\varphi_{n}}=\alpha_{\varphi_{n}}=1$ and $\lambda(\Gamma)=\Gamma^{+}=\Gamma \backslash\{1, \ldots, n-1\}$.

For every $n \in \mathbb{N}$, let $\psi_{n}: \Gamma \rightarrow \Gamma$ be defined by

$$
\psi_{n}(m)= \begin{cases}0 & \text { if } m=0,1, \ldots, n, \\ (k-1) n+i & \text { if } m=k n+i \text { with } 0 \leq i<n \text { and } k \in \mathbb{N} .\end{cases}
$$

The diagram for $\psi_{n}$ is the following:

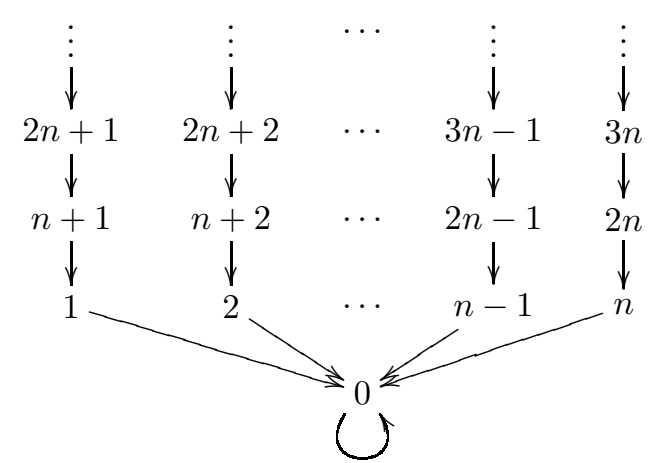

For this function $s_{\psi_{n}}=n, \alpha_{\psi_{n}}=1$ and $\Gamma^{+}=\Gamma$. Note that $\psi_{1}=\varphi_{1}$.

Let $\Gamma=\mathbb{N}_{0} \times \mathbb{N}_{0}$ and $\lambda_{0}: \Gamma \rightarrow \Gamma$ be defined by

$$
\lambda_{0}(m, k)= \begin{cases}(0,0) & \text { if } m=k=0 \\ (m-1,0) & \text { if } k=0 \text { and } m \in \mathbb{N} \\ (m, k-1) & \text { if } m \in \mathbb{N}_{0} \text { and } k \in \mathbb{N}\end{cases}
$$

The diagram for $\lambda_{0}$ is the following:

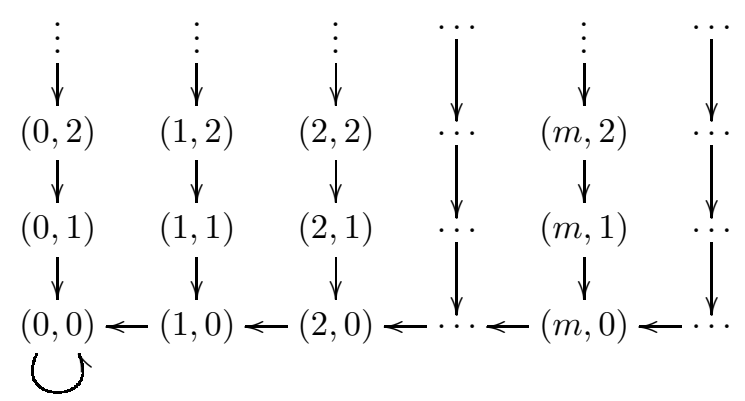

In this case $s_{\lambda_{0}}=\omega, \alpha_{\lambda_{0}}=1$ and $\Gamma^{+}=\Gamma$. 


\section{The ENtropy of the Generalized SHIFT}

Remark 4.1. Let $\mu: \Gamma \rightarrow \Gamma$ be a function. If $H$ is a subgroup of an abelian group $L$, then $H^{\Gamma}$ is a $\sigma_{\mu, L}$-invariant subgroup of $L^{\Gamma}$. Moreover, $\sigma_{\mu, L} \uparrow_{H^{\Gamma}}=\sigma_{\mu, H}: H^{\Gamma} \rightarrow H^{\Gamma}$. Analogously, if $L^{(\Gamma)}$ is a $\sigma_{\mu, L}^{\oplus}$-invariant subgroup of $L^{\Gamma}$, then $H^{(\Gamma)}$ is a $\sigma_{\mu, L^{\oplus}}^{\oplus}$-invariant subgroup of $L^{(\Gamma)}$, and $\sigma_{\mu, L}^{\oplus} \uparrow_{H^{(\Gamma)}}=\sigma_{\mu, H}^{\oplus}$ : $H^{(\Gamma)} \rightarrow H^{(\Gamma)}$.

Claim 4.2. Let $x \in G_{\Gamma}$ and $F=\operatorname{supp}(x)$. Then for every $m \in \mathbb{N}$ :

(a) $\operatorname{supp}\left(\sigma_{\lambda}^{m}(x)\right)=\lambda^{-m}(F)$;

(b) $\sigma_{\lambda}^{m}\left(G_{F}\right) \leq G_{\lambda^{-m}(F)}$;

(c) $T_{m}\left(\sigma_{\lambda}, G_{F}\right) \leq G_{F \cup \lambda^{-1}(F) \cup \ldots \cup \lambda^{-m+1}(F)}$.

Proof. (a) If $y=\sigma_{\lambda}(x)$, then $y_{i}=x_{\lambda(i)} \neq 0$ if and only if $\lambda(i) \in F$, that is, $i \in \lambda^{-1}(F)$, and so $\operatorname{supp}(y)=\lambda^{-1}(F)$. Proceeding by induction it is possible to prove that $\operatorname{supp}\left(\sigma_{\lambda}^{m}(x)\right)=\lambda^{-m}(F)$ for every $m \in \mathbb{N}$.

(b) Follows from (a).

(c) By (b) $T_{m}\left(\sigma_{\lambda}, G_{F}\right) \leq G_{F}+G_{\lambda^{-1}(F)}+\ldots+G_{\lambda^{-m+1}(F)}$ and $G_{F}+G_{\lambda^{-1}(F)}+\ldots+G_{\lambda^{-m+1}(F)} \subseteq$ $G_{F \cup \lambda^{-1}(F) \cup \ldots \cup \lambda^{-m+1}(F)}$.

The next lemma shows the relevance of our following assumption on $\lambda$ of having finite fibers.

Lemma 4.3. The following conditions are equivalent:

(a) $\lambda^{-1}(i)$ is finite for each $i \in \Gamma$;

(b) $\sigma_{\lambda, K}\left(G_{\Gamma}\right) \subseteq G_{\Gamma}$;

(c) $\sigma_{\lambda, L}\left(L^{(\Gamma)}\right) \subseteq L^{(\Gamma)}$ for every non-trivial abelian group $L$.

Proof. (b) $\Rightarrow(\mathrm{a})$ Let $i \in \Gamma$ and $x \in G_{\{i\}} \backslash\{0\}$. By Claim 4.2(a) $\operatorname{supp}\left(\sigma_{\lambda}(x)\right)=\lambda^{-1}(i)$ and by the assumption $\sigma_{\lambda}(x) \in G_{\Gamma}$, hence $\lambda^{-1}(i)$ is finite.

A similar argument shows that $(\mathrm{a}) \Rightarrow(\mathrm{c})$ and $(\mathrm{c}) \Rightarrow(\mathrm{b})$ is obvious.

Convention. From now on we suppose that $\lambda$ has finite fibers, that is, $\lambda^{-1}(i)$ is finite for every $i \in \Gamma$.

Proposition 4.4. Let $L$ be an abelian group with at least two elements and let $\mu, \nu: \Gamma \rightarrow \Gamma$ be functions with finite fibers. For $\sigma_{\mu}, \sigma_{\nu}: L^{\Gamma} \rightarrow L^{\Gamma}$ and $\sigma_{\mu}^{\oplus}, \sigma_{\nu}^{\oplus}: L^{(\Gamma)} \rightarrow L^{(\Gamma)}$ :

(a) $\sigma_{\mu} \circ \sigma_{\nu}=\sigma_{\mu \circ \nu}$ and $\sigma_{\mu}^{\oplus} \circ \sigma_{\nu}^{\oplus}=\sigma_{\mu \circ \nu}^{\oplus}$ (hence $\sigma_{\mu}^{m}=\sigma_{\mu^{m}}$ and $\left(\sigma_{\mu}^{\oplus}\right)^{m}=\sigma_{\mu^{m}}^{\oplus}$ for every $\left.m \in \mathbb{N}\right)$.

(b) 3] The following conditions are equivalent:

$\left(\mathrm{b}_{1}\right) \mu$ is injective (respectively, surjective);

$\left(\mathrm{b}_{2}\right) \sigma_{\mu}$ is surjective (respectively, injective);

$\left(\mathrm{b}_{3}\right) \sigma_{\mu}^{\oplus}$ is surjective (respectively, injective).

(c) In particular, the following conditions are equivalent:

$\left(\mathrm{c}_{1}\right) \mu$ is bijective;

$\left(\mathrm{c}_{2}\right) \sigma_{\mu}$ is an automorphism of $L^{\Gamma}$;

$\left(\mathrm{c}_{3}\right) \sigma_{\mu}^{\oplus}$ is an automorphism of $L^{(\Gamma)}$.

In this case, $\left(\sigma_{\mu}\right)^{-1}=\sigma_{\mu^{-1}}$ and $\left(\sigma_{\mu}^{\oplus}\right)^{-1}=\sigma_{\mu^{-1}}^{\oplus}$.

Note that the equivalences $\left(b_{1}\right) \Leftrightarrow\left(b_{2}\right)$ and $\left(c_{1}\right) \Leftrightarrow\left(c_{2}\right)$ hold without any assumption on the fibers of $\mu$ and $\nu$.

Corollary 4.5. For every $m \in \mathbb{N}$, $\operatorname{ker} \sigma_{\lambda}^{m}=G_{\Gamma \backslash \lambda^{m}(\Gamma)}$.

Proof. It suffices to prove that $\operatorname{ker} \sigma_{\lambda}=G_{\Gamma \backslash \lambda(\Gamma)}$ and then apply Proposition 4.4(a). If $x \in$ ker $\sigma_{\lambda}$, equivalently $\operatorname{supp}\left(\sigma_{\lambda}(x)\right)=\emptyset$. By Claim $4.2(\mathrm{a}) \operatorname{supp}\left(\sigma_{\lambda}(x)\right)=\lambda^{-1}(\operatorname{supp}(x))$. Then $\operatorname{supp}\left(\sigma_{\lambda}(x)\right)=$ $\lambda^{-1}(\operatorname{supp}(x))=\emptyset$ if and only if $\operatorname{supp}(x) \cap \lambda(\Gamma)=\emptyset$. This is the same as $\operatorname{supp}(x) \subseteq \Gamma \backslash \lambda(\Gamma)$, that is, $x \in G_{\Gamma \backslash \lambda(\Gamma)}$.

The next lemma gives a characterization (in terms of $\lambda$ ) of the $\sigma_{\lambda}$-invariance of the subgroups $G_{A}$ of $G_{\Gamma}$. 
Lemma 4.6. If $A \subseteq \Gamma$, then $G_{A}$ is $\sigma_{\lambda}$-invariant if and only if $\lambda^{-1}(A) \subseteq A$. If $A$ is also $\lambda$-invariant, then $\sigma_{\lambda} \uparrow_{G_{A}}=\sigma_{\lambda \uparrow_{A}}$.

Proof. The condition $\sigma_{\lambda}\left(G_{A}\right) \subseteq G_{A}$ is equivalent to $\sigma_{\lambda}\left(G_{i}\right) \subseteq G_{A}$ for every $i \in A$, that is, $\lambda^{-1}(i) \subseteq A$ for every $i \in A$, which is equivalent to $\lambda^{-1}(A) \subseteq A$. Assume now that $\lambda^{-1}(A) \cup \lambda(A) \subseteq A$. Then it is possible to consider both $\sigma_{\lambda} \uparrow_{G_{A}}$ and $\sigma_{\lambda \uparrow_{A}}$. It is clear that they coincide on $G_{A}$.

Lemma 4.6 shows that in case $\lambda^{-1}(A) \subseteq A$ for $A \subseteq \Gamma$, it is possible to consider $\sigma_{\lambda} \Upsilon_{G_{A}}: G_{A} \rightarrow G_{A}$.

Remark 4.7. We see here that we can assume that for the relation $\Re_{\lambda}$ there exists only one equivalence class in $\Gamma$ (so coinciding with the whole $\Gamma$ ).

Indeed, if $i / \Re_{\lambda}$ is a generic equivalence class, then $i / \Re_{\lambda} \supseteq \lambda\left(i / \Re_{\lambda}\right) \cup \lambda^{-1}\left(i / \Re_{\lambda}\right)$. By Lemma 4.6

$$
\sigma_{\lambda} \uparrow_{i / \Re_{\lambda}}=\sigma_{\lambda \uparrow_{i / \Re_{\lambda}}} \text {. }
$$

Let now $R$ be a set of representing elements of $\Re_{\lambda}$ in $\Gamma$. Then $G_{\Gamma}=\bigoplus_{i \in R} G_{i / \Re_{\lambda}}$. By Remark 2.4(c) and (4.1)

$$
\operatorname{ent}\left(\sigma_{\lambda}\right)=\sum_{i \in R} \operatorname{ent}\left(\sigma_{\lambda}\left\lceil_{G_{i / \Re_{\lambda}}}\right)=\sum_{i \in R} \operatorname{ent}\left(\sigma_{\lambda \uparrow_{i / \Re_{\lambda}}}\right),\right.
$$

and so we can assume that $R$ is a singleton.

The next result gives the very useful formula (4.2), which is applied in the proof of the main theorem.

Remark 4.8. Let $\Gamma=\Gamma^{\prime} \cup \Gamma^{\prime \prime}$ be a partition of $\Gamma$. Then $\lambda^{-1}\left(\Gamma^{\prime}\right) \subseteq \Gamma^{\prime}$ if and only if $\lambda\left(\Gamma^{\prime \prime}\right) \subseteq \Gamma^{\prime \prime}$. Suppose that these equivalent conditions hold. By Lemma $4.6 G_{\Gamma^{\prime}}$ is $\sigma_{\lambda}$-invariant.

(a) Let $p_{2}: G_{\Gamma}=G_{\Gamma^{\prime}} \oplus G_{\Gamma^{\prime \prime}} \rightarrow G_{\Gamma^{\prime \prime}}$ and $\pi: G_{\Gamma} \rightarrow G_{\Gamma} / G_{\Gamma^{\prime}}$ be the canonical projections. Denote by $\xi: G_{\Gamma} / G_{\Gamma^{\prime}} \rightarrow G_{\Gamma^{\prime \prime}}$ the (unique) isomorphism such that $p_{2}=\xi \circ \pi$. Finally, let $\overline{\sigma_{\lambda}}: G_{\Gamma} / G_{\Gamma^{\prime}} \rightarrow G_{\Gamma} / G_{\Gamma^{\prime}}$ be the homomorphism induced by $\sigma_{\lambda}$. Then $\overline{\sigma_{\lambda}}=\xi^{-1} \sigma_{\lambda \Gamma_{\Gamma^{\prime \prime}}} \xi$. To better explain the situation, this means that the following diagram commutes:

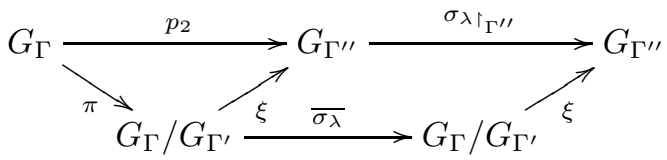

By Lemma $2.1 \operatorname{ent}\left(\overline{\sigma_{\lambda}}\right)=\operatorname{ent}\left(\sigma_{\lambda \Gamma_{\Gamma^{\prime \prime}}}\right)$.

(b) By (a) and Theorem 2.3

$$
\operatorname{ent}\left(\sigma_{\lambda}\right)=\operatorname{ent}\left(\sigma_{\lambda} \uparrow_{G_{\Gamma^{\prime}}}\right)+\operatorname{ent}\left(\sigma_{\lambda \uparrow_{\Gamma^{\prime \prime}}}\right)
$$

Therefore,

The next corollary shows that ent $\left(\sigma_{\lambda}\right)$ can be computed from its restriction to $\Gamma^{+} \subseteq \Gamma$.

\section{Corollary 4.9. (a) $\operatorname{ent}\left(\sigma_{\lambda}\right)=\operatorname{ent}\left(\sigma_{\lambda \uparrow_{\Gamma^{+}}}\right)$.}

(b) In particular, for each $k \in \mathbb{N}$, ent $\left(\sigma_{\lambda}\right)=\operatorname{ent}\left(\sigma_{\lambda_{\Gamma_{\lambda} k}(\Gamma)}\right)$.

(c) If there exists a non-empty finite subset $F$ of $\Gamma$ such that $\Gamma=\bigcup_{n \in \mathbb{N}_{0}} \lambda^{n}(F)$, then $\Gamma^{+}=\operatorname{Per}(\lambda)$ and consequently $\operatorname{ent}\left(\sigma_{\lambda}\right)=0$.

Proof. (a) Since $\lambda\left(\Gamma^{+}\right) \subseteq \Gamma^{+}$, by (4.2) in Remark 4.8(b) $\operatorname{ent}\left(\sigma_{\lambda}\right)=\operatorname{ent}\left(\sigma_{\lambda} \uparrow_{G_{\Gamma \Gamma^{+}}}\right)+\operatorname{ent}\left(\sigma_{\lambda \Gamma_{\Gamma^{+}}}\right)$. We prove that $\operatorname{ent}\left(\sigma_{\lambda}\left\lceil_{G_{\Gamma \backslash \Gamma^{+}}}\right)=0\right.$. Let $x \in G_{\Gamma \backslash \Gamma^{+}}$. Then $\operatorname{supp}(x) \subseteq \Gamma \backslash \Gamma^{+}$. For every $i \in \operatorname{supp}(x)$ there exists $h(i) \in \mathbb{N}$ such that $i \notin \lambda^{h(i)}(\Gamma)$, and so $\lambda^{-h(i)}(i)=\emptyset$. Let $h(x)=\max \{h(i): i \in \operatorname{supp}(x)\}$. By Claim 4.2(a) $\operatorname{supp}\left(\sigma_{\lambda}^{h(x)}(x)\right)=\lambda^{-h(x)}(\operatorname{supp}(x))$, which is empty, and so $\sigma_{\lambda}^{h(x)}(x)=0$. By Lemma 2.5) (a) $\operatorname{ent}\left(\sigma_{\lambda}\left\lceil_{G_{\Gamma \backslash \Gamma^{+}}}\right)=0\right.$.

(b) Follows from (a) since $\operatorname{ent}\left(\sigma_{\lambda}\right) \geq \operatorname{ent}\left(\sigma_{\lambda_{\lambda^{k}(\Gamma)}}\right) \geq \operatorname{ent}\left(\sigma_{\lambda_{\Gamma^{+}}+}\right)$, where (4.3) in Remark 4.8(b) is applied twice.

(c) Clearly $\Gamma^{+} \supseteq \operatorname{Per}(\lambda)$. Let $i \in \Gamma^{+}$. For every $n \in \mathbb{N}$ there exists $i_{n} \in \Gamma$ such that $\lambda^{n}\left(i_{n}\right)=i$. Moreover, for $n \in \overline{\mathbb{N}}$ there exist $m_{n} \in \mathbb{N}$ and $j_{m_{n}} \in F$ such that $\lambda^{m_{n}}\left(j_{m_{n}}\right)=i_{n}$ and so $\lambda^{n+m_{n}}\left(j_{m_{n}}\right)=i$. Since $F$ is finite, there exists $j \in F$ such that for a strictly increasing sequence $\left(n_{k}\right)_{k \in \mathbb{N}}$ in $\mathbb{N}$ one has $j_{m_{n_{k}}}=j$ for all $k \in \mathbb{N}$. Then $\lambda^{n_{k}+m_{n_{k}}}(j)=i$ for all $k \in \mathbb{N}$. Choose $k \in \mathbb{N}$ such that $n_{k}>n_{1}+m_{n_{1}}$, then $n_{k}+m_{n_{k}}>n_{1}+m_{n_{1}}$ as well. Therefore, $i=\lambda^{n_{1}+m_{n_{1}}}(j)=\lambda^{n_{k}+m_{n_{k}}}(j)$ yields $i \in \operatorname{Per}(\lambda)$. 
Example 4.10. Let $\lambda: \mathbb{N}_{0} \rightarrow \mathbb{N}_{0}$ be defined by $\lambda(n)=n+1$. Then $\sigma_{\lambda}: K^{\left(\mathbb{N}_{0}\right)} \rightarrow K^{\left(\mathbb{N}_{0}\right)}$ coincides with the left Bernoulli shift ${ }_{K} \beta$ of $K^{\left(\mathbb{N}_{0}\right)}$. Since $\mathbb{N}_{0}^{+}=\emptyset$ (in the notation of Corollary 4.9), we deduce from Corollary 4.9. (c) that ent $\left(\sigma_{\lambda}\right)=0$.

Another application of Corollary 4.9 is the following example, in which $\Gamma$ is a compact metric space.

Example 4.11. Let $(\Gamma, d)$ be a compact metric space and $\lambda: \Gamma \rightarrow \Gamma$ be a contraction (i.e., $d(\lambda(x), \lambda(y))<$ $d(x, y)$ for every pair of distinct points $x, y$ of $\Gamma)$ such that for each $i \in \Gamma, \lambda^{-1}(i)$ is finite. Then $\operatorname{ent}\left(\sigma_{\lambda}\right)=0$.

Indeed, using a standard compactness argument one proves that $\lambda\left(\Gamma^{+}\right)=\Gamma^{+} \neq \emptyset$. Moreover, $\left|\Gamma^{+}\right|=1$ since otherwise there exist $x, y \in \Gamma^{+}$such that $d(x, y)=\operatorname{diam} \Gamma^{+}$. By $\lambda\left(\Gamma^{+}\right)=\Gamma^{+}$there exist $x^{\prime}, y^{\prime} \in \Gamma^{+}$with $\lambda\left(x^{\prime}\right)=x$ and $\lambda\left(y^{\prime}\right)=y$. So diam $\Gamma^{+}=d(x, y)=d\left(\lambda\left(x^{\prime}\right), \lambda\left(y^{\prime}\right)\right)<d\left(x^{\prime}, y^{\prime}\right)$, a contradiction. Therefore, $\left|\Gamma^{+}\right|=1$, so Corollary 4.9(a) applies.

4.1. The main theorem. For a set $X$, in what follows we use the following notation:

$$
|X|^{*}:= \begin{cases}|X| & \text { in case }|X| \text { is finite, } \\ +\infty & \text { in case }|X| \text { is infinite. }\end{cases}
$$

Definition 4.12. Two strings $S$ and $S^{\prime}$ of $\lambda$ in the set $\Gamma$ are said to be strongly disjoint if $S$ and $\lambda^{n}\left(S^{\prime}\right)$ are disjoint for every $n \in \mathbb{N}_{0}$ and $S^{\prime}$ and $\lambda^{n}(S)$ are disjoint for every $n \in \mathbb{N}_{0}$.

By the definition of string we immediately have the following result.

Claim 4.13. If $s_{\lambda}=n \in \mathbb{N}_{0}$ is finite, then $\Gamma$ contains $n$ strings of $\lambda$ that are pairwise strongly disjoint.

The next is our main theorem, which calculates the entropy of a generalized shift $\sigma_{\lambda}: G_{\Gamma} \rightarrow G_{\Gamma}$, proving that it depends only on the function $\lambda: \Gamma \rightarrow \Gamma$ and on the cardinality of $K$.

Theorem 4.14. Let $\Gamma$ be a set, $\lambda: \Gamma \rightarrow \Gamma$ a function such that $\lambda^{-1}(i)$ is finite for every $i \in \Gamma$, and consider $\sigma_{\lambda}: G_{\Gamma} \rightarrow G_{\Gamma}$, where $G_{\Gamma}=K^{(\Gamma)}$ and $K$ is a non-trivial finite abelian group. Then

$$
\operatorname{ent}\left(\sigma_{\lambda}\right)=\left|s_{\lambda}\right|^{*} \log |K| \text {. }
$$

Proof. By Corollary 4.9(a) and Remark 4.7 we can assume without loss of generality that $\Gamma=\Gamma^{+}$and that there exists only one equivalence class for $\Re_{\lambda}$.

Suppose that $s_{\lambda}=n$ for some $n \in \mathbb{N}_{0}$. If $n>0$, there exist $n$ pairwise strongly disjoint acyclic strings of $\lambda$

in $\Gamma$ by Claims 3.2 and 4.13 ,

$$
S_{1}:=\left\{m_{t}^{1}\right\}_{-t \in \mathbb{N}_{0}}, \ldots, S_{n}:=\left\{m_{t}^{n}\right\}_{-t \in \mathbb{N}_{0}}
$$

Let $\Psi=\emptyset$ if $n=0$ and $\Psi:=S_{1} \cup \ldots \cup S_{n}$ otherwise. Then $\lambda^{-1}(\Psi) \subseteq \Psi$, since $\Gamma=\Gamma^{+}$, and so $G_{\Psi}$ is $\sigma_{\lambda}$-invariant by Lemma 4.6 Let $\nu:=\lambda \Gamma_{\Gamma \backslash \Psi}$. By (4.2) in Remark 4.8(b)

$$
\operatorname{ent}\left(\sigma_{\lambda}\right)=\operatorname{ent}\left(\sigma_{\lambda}\left\lceil_{G_{\Psi}}\right)+\operatorname{ent}\left(\sigma_{\nu}\right)\right.
$$

For $F=\left\{\lambda\left(m_{0}^{1}\right), \ldots, \lambda\left(m_{0}^{n}\right)\right\}, \Gamma \backslash \Psi=\bigcup_{n \in \mathbb{N}_{0}} \nu^{n}(F)$, since the strings are acyclic. By Corollary 4.9 (c) $\operatorname{ent}\left(\sigma_{\nu}\right)=0$ and so

$$
\operatorname{ent}\left(\sigma_{\lambda}\right)=\operatorname{ent}\left(\sigma_{\lambda}\left\lceil_{G_{\Psi}}\right) .\right.
$$

Now $\Psi$ is disjoint union of $S_{1}, \ldots, S_{n}$ and so $G_{\Psi}=G_{S_{1}} \oplus \ldots \oplus G_{S_{n}}$. Moreover, $\lambda^{-1}\left(S_{j}\right) \subseteq S_{j}$ since $\Gamma=\Gamma^{+}$, and so by Lemma 4.6 $G_{S_{j}}$ is $\sigma_{\lambda}$-invariant for each $j \in\{1, \ldots, n\}$. By Remark 2.4(b)

$$
\operatorname{ent}\left(\sigma_{\lambda}\left\lceil_{G_{\Psi}}\right)=\operatorname{ent}\left(\sigma_{\lambda}\left\lceil_{G_{S_{1}}}\right)+\ldots+\operatorname{ent}\left(\sigma_{\lambda}\left\lceil_{G_{S_{n}}}\right)\right. \text {. }\right.\right.
$$

For every $j \in\{1, \ldots, n\}$, since $\sigma_{\lambda}\left\lceil_{G_{S_{j}}}\right.$ is precisely the right Bernoulli shift $\beta_{K} \uparrow_{G_{S_{j}}}: G_{S_{j}} \rightarrow G_{S_{j}}$,

$$
\operatorname{ent}\left(\sigma_{\lambda}\left\lceil_{G_{S_{j}}}\right)=\log |K|\right. \text {. }
$$

By (4.4), (4.5) and (4.6) ent $\left(\sigma_{\lambda}\right)=n \log |K|=s_{\lambda} \log |K|$.

Assume now that $\left|s_{\lambda}\right|^{*}=+\infty$. Then $s_{\lambda}>n$ for every $n \in \mathbb{N}$. Fix $n \in \mathbb{N}$. There exist $n$ pairwise disjoint strings of $\lambda$

$$
S_{1}:=\left\{m_{t}^{1}\right\}_{-t \in \mathbb{N}_{0}}, \ldots, S_{n}:=\left\{m_{t}^{n}\right\}_{-t \in \mathbb{N}_{0}}
$$


in $\Gamma$. Define

$$
\Lambda_{1}:=S_{1} \cup\left\{\lambda^{s}\left(m_{0}^{1}\right): s \in \mathbb{N}\right\}, \ldots, \Lambda_{n}:=S_{n} \cup\left\{\lambda^{s}\left(m_{0}^{n}\right): s \in \mathbb{N}\right\} \text { and } \Lambda:=\Lambda_{1} \cup \ldots \cup \Lambda_{n} .
$$

Note that $\lambda(\Lambda) \subseteq \Lambda$, so that we can consider the map $\left.\lambda\right|_{\Lambda}: \Lambda \rightarrow \Lambda$. By (4.3) in Remark 4.8(b)

$$
\operatorname{ent}\left(\sigma_{\lambda}\right) \geq \operatorname{ent}\left(\sigma_{\left.\lambda\right|_{\Lambda}}\right) \text {. }
$$

Since $s_{\left.\lambda\right|_{\Lambda}}=n$, by the finite case of the proof of the theorem applied to the map $\lambda\left\lceil_{\Lambda}\right.$, ent $\left(\sigma_{\left.\lambda\right|_{\Lambda}}\right)=$ $n \log |K|$. By (4.7) $\operatorname{ent}\left(\sigma_{\lambda}\right) \geq n \log |K|$, and this holds true for every $n \in \mathbb{N}$, so that $\operatorname{ent}\left(\sigma_{\lambda}\right)=+\infty=$ $\left|s_{\lambda}\right|^{*} \log |K|$.

We see now a first application of our main theorem.

Example 4.15. (a) In general ent $\left(\sigma_{\lambda}\right) \geq\left|\alpha_{\lambda}\right|^{*} \log |K|$. Indeed, by the definitions $s_{\lambda} \geq \alpha_{\lambda}$ and so apply Theorem 4.14

(b) The inequality in (a) can be strict: consider the functions of Example 3.4. In all three cases there exists just one equivalence class and so one equivalent class containing at least one infinite string of $\lambda$. Then $\alpha_{\varphi_{n}}=\alpha_{\psi_{n}}=\alpha_{\lambda_{0}}=1$, but ent $\left(\sigma_{\varphi_{n}}\right)=\log |K|$, ent $\left(\sigma_{\psi_{n}}\right)=n \log |K|$ and $\operatorname{ent}\left(\sigma_{\lambda_{0}}\right)=+\infty$.

(c) If $\lambda: \Gamma \rightarrow \Gamma$ is injective, the inequality in (a) becomes an equality, since in this case $\alpha_{\lambda}=s_{\lambda}$ : by Theorem 4.14 and Example $3.3 \operatorname{ent}\left(\sigma_{\lambda}\right)=\left|\alpha_{\lambda}\right|^{*} \log |K|=\left|s_{\lambda}\right|^{*} \log |K|$.

(d) For $\lambda: \mathbb{Z} \rightarrow \mathbb{Z}$ defined by $\lambda(n)=n-1$ for every $n \in \mathbb{Z}$, the generalized shift $\sigma_{\lambda}$ coincides with the two-sided shift $\bar{\beta}_{K} \uparrow_{K^{(\mathbb{Z})}}$ of $K^{(\mathbb{Z})}$. Since $\alpha_{\lambda}=s_{\lambda}=1$, one obtains from Theorem 4.14 $\operatorname{ent}\left(\bar{\beta}_{K} \uparrow_{K}^{(\mathbb{Z})}\right)=\operatorname{ent}\left(\sigma_{\lambda}\right)=\log |K|$.

\section{Applications of the main theorem}

We give now other consequences of Theorem 4.14. The first one is an application of Theorem 4.14 together with Remark 4.8(b). It shows that, even if the restriction of $\sigma_{\lambda}$ to an invariant subgroup is not necessarily a generalized shift, its entropy obeys the same formula as the generalized shift does.

Corollary 5.1. Let $\Lambda \subseteq \Gamma$ be such that $\lambda^{-1}(\Lambda) \subseteq \Lambda$. Then $\left|s_{\lambda}\right|^{*} \log |K|=\operatorname{ent}\left(\sigma_{\lambda}\left\lceil_{G_{\Lambda}}\right)+\left|s_{\left.\lambda\right|_{\Gamma \backslash \Lambda}}\right|{ }^{*} \log |K|\right.$.

Proof. By (4.2) in Remark4.8(b) and Theorem 4.14 $\left|s_{\lambda}\right|^{*} \log |K|=\operatorname{ent}\left(\sigma_{\lambda}\right)=\operatorname{ent}\left(\sigma_{\lambda}\left\lceil G_{\Lambda}\right)+\operatorname{ent}\left(\sigma_{\left.\lambda\right|_{\Gamma \backslash \Lambda}}\right)=\right.$ $\operatorname{ent}\left(\sigma_{\lambda}\left\lceil_{G_{\Lambda}}\right)+\left|s_{\lambda \Gamma_{\Gamma \backslash \Lambda} \mid}{ }^{*} \log \right| K \mid\right.$.

Remark 5.2. If $\Lambda \subseteq \Gamma$ is such that $\lambda^{-1}(\Lambda) \subseteq \Lambda$, and $S$ is a string of $\lambda$ with $S \cap \Lambda \neq \emptyset$, then $S \backslash \Lambda$ is finite and we can assume without loss of generality that $S \subseteq \Lambda$. Hence we can think that either $S \subseteq \Lambda$ or $S \subseteq \Gamma \backslash \Lambda$. This shows that:

(a) $s_{\left.\lambda\right|_{\Gamma \backslash \Lambda}}$ is the number of all strings of $\lambda$ which miss $\Lambda$, and

(b) in case $s_{\lambda}$ is finite, $s_{\lambda}-s_{\lambda_{\Gamma \backslash \Lambda}}$ is the number of all strings of $\lambda$ contained in $\Lambda$.

Corollary 5.3. (a) Let $\Lambda \subseteq \Gamma$ be such that $\lambda^{-1}(\Lambda) \subseteq \Lambda$. If $\left\{\operatorname{ent}\left(\sigma_{\lambda}\right)\right.$, $\operatorname{ent}\left(\sigma_{\lambda}\left\lceil G_{\Lambda}\right)\right\} \cap\{0,+\infty\}=\emptyset$, then $\operatorname{ent}\left(\sigma_{\lambda} \Gamma_{G_{\Lambda}}\right) / \operatorname{ent}\left(\sigma_{\lambda}\right)$ is rational. Moreover, if $0<r \leq 1$ is a rational number such that $r\left(\operatorname{ent}\left(\sigma_{\lambda}\right) / \log |K|\right) \in \mathbb{Z}$, then there exists $\Lambda \subseteq \Gamma$, such that $\operatorname{ent}\left(\sigma_{\lambda}\left\lceil G_{\Lambda}\right) / \operatorname{ent}\left(\sigma_{\lambda}\right)=r\right.$.

(b) Let $L$ be another finite abelian group and assume that both $K$ and $L$ have at least two elements. Then $\log |L| \operatorname{ent}\left(\sigma_{\lambda, K}\right)=\log |K| \operatorname{ent}\left(\sigma_{\lambda, L}\right)$.

Proof. (a) By Theorem 4.14, Corollary 5.1 and our assumption $\left\{\operatorname{ent}\left(\sigma_{\lambda}\right), \operatorname{ent}\left(\sigma_{\lambda}\left\lceil_{G_{\Lambda}}\right)\right\} \cap\{0,+\infty\}=\emptyset\right.$, $s_{\lambda}$ is finite and $\operatorname{ent}\left(\sigma_{\lambda} \Gamma_{G_{\Lambda}}\right) / \operatorname{ent}\left(\sigma_{\lambda}\right)=\left(s_{\lambda}-s_{\lambda_{\Gamma \backslash \Lambda}}\right) / s_{\lambda}$ is a rational number.

By hypothesis there exists $m \in \mathbb{N}$ such that $r s_{\lambda}=m$. Let $S_{1}, \ldots, S_{s_{\lambda}}$ be the strongly disjoint strings in $\Gamma$ that realize $s_{\lambda}$ (this is possible by Claim 4.13 since $s_{\lambda}$ is finite by hypothesis). Since $r \leq 1$, it follows that $m \leq s_{\lambda}$. So it is possible to consider $T:=S_{1} \cup \ldots \cup S_{m}$ and define $\Lambda:=\bigcup_{n \in \mathbb{N}_{0}} \lambda^{-n}(T)$. Then $\lambda^{-1}(\Lambda) \subseteq \Lambda$. By Remark $5.2 s_{\lambda}-s_{\left.\lambda\right|_{\Gamma \backslash \Lambda}}=m$, since $\left\{S_{1}, \ldots, S_{m}\right\}$ is a family of pairwise disjoint strings in $\Lambda$ of the maximal possible cardinality. By Corollary $5.1 \operatorname{ent}\left(\sigma_{\lambda}\left\lceil_{G_{\Lambda}}\right)=m \log |K|\right.$. Then $\operatorname{ent}\left(\sigma_{\lambda}\left\lceil_{G_{\Lambda}}\right) / \operatorname{ent}\left(\sigma_{\lambda}\right)=m / s_{\lambda}=r\right.$.

(b) Is a simple application of Theorem 4.14 . 
Corollary 5.4. For $M$ a torsion infinite abelian group and $\sigma_{\lambda}: M^{(\Gamma)} \rightarrow M^{(\Gamma)}$,

$$
\operatorname{ent}\left(\sigma_{\lambda}\right)= \begin{cases}0 & \text { if } s_{\lambda}=0 \\ +\infty & \text { if } s_{\lambda}>0\end{cases}
$$

Proof. In view of Remark 4.1 and Theorem 4.14, it is easy to see that

$$
\begin{aligned}
\operatorname{ent}\left(\sigma_{\lambda}\right) & =\sup \left\{\operatorname{ent}\left(\sigma_{\lambda} \uparrow_{H^{(\Gamma)}}\right): H \text { is a finite subgroup of } M\right\} \\
& =\sup \left\{\operatorname{ent}\left(\sigma_{\lambda, H}\right): H \text { is a finite subgroup of } M\right\} \\
& =\sup \left\{\left|s_{\lambda}\right|^{*} \log |H|: H \text { is a finite subgroup of } M\right\}
\end{aligned}
$$

If $s_{\lambda}=0, \operatorname{ent}\left(\sigma_{\lambda}\right)=0$. If $s_{\lambda}>0$, then $\operatorname{ent}\left(\sigma_{\lambda}\right)$ converges to $+\infty$ with $\log |H|$.

Example 5.5. (a) Let $\Gamma$ be a monoid.

$\left(\mathrm{a}_{1}\right)$ For each $s \in \Gamma$ consider $\lambda_{s}, \rho_{s}: \Gamma \rightarrow \Gamma$ defined by $\lambda_{s}(t)=s t$ and $\rho_{s}(t)=t s$ for every $t \in \Gamma$. The element $s$ is invertible if and only if $\lambda_{s}$ and $\rho_{s}$ are bijective. By Example 4.15, the endomorphisms $\sigma_{\lambda_{s}}$ and $\sigma_{\rho_{s}}$ of $G_{\Gamma}$ have the following properties.

(i) If $s$ is of finite order $n \in \mathbb{N}$, then $\left(\lambda_{s}\right)^{n}=\lambda_{s^{n}}=i d_{\Gamma}$ (i.e., $\lambda_{s}$ is periodic) and similarly $\left(\rho_{s}\right)^{n}=\rho_{s^{n}}=i d_{\Gamma}$ (i.e., $\rho_{s}$ is periodic). By Proposition 4.4 (a) $\sigma_{\lambda_{s}}^{n}=\sigma_{\lambda_{s}^{n}}=\sigma_{\lambda_{s} n}=$ $i d_{G_{\Gamma}}$ and $\sigma_{\rho_{s}}^{n}=\sigma_{\rho_{s}^{n}}=\sigma_{\rho_{s} n}=i d_{G_{\Gamma}}$. Hence $\operatorname{ent}\left(\sigma_{\lambda_{s}}\right)=\operatorname{ent}\left(\sigma_{\rho_{s}}\right)=0$ by Lemma $2.5(\mathrm{~b})$.

(ii) Suppose that $s$ is invertible. If $s$ has infinite order, then $\lambda_{s}$ and $\rho_{s}$ are injective and by Example 4.15 their entropy is positive. So, $\operatorname{ent}\left(\sigma_{\lambda_{s}}\right)=\operatorname{ent}\left(\sigma_{\rho_{s}}\right)=0$ if and only if $s$ is of finite order.

$\left(\mathrm{a}_{2}\right)$ For each invertible $s \in \Gamma$ consider $\mu_{s}: \Gamma \rightarrow \Gamma$ defined by $\mu_{s}(t)=s^{-1}$ for every $t \in \Gamma$. By Example 4.15, the endomorphism $\sigma_{\mu_{s}}$ of $G_{\Gamma}$ has ent $\left(\sigma_{\mu_{s}}\right)>0$ if and only if there exists $t \in \Gamma$ such that $\left\{s^{n}: n \in \mathbb{N}\right\} \cap\{v \in \Gamma: v t=t v\}=\emptyset$.

(b) Suppose now that $\Gamma$ is an abelian group and $\lambda: \Gamma \rightarrow \Gamma$ a group homomorphism such that ker $\lambda$ is finite (i.e., $\lambda$ has finite fibers).

$\left(\mathrm{b}_{1}\right)$ If $\Gamma=\mathbb{Z}$, there exists $n \in \mathbb{Z}$ such that $\lambda(x)=n x$ for every $x \in \mathbb{Z}$. If $n \neq \pm 1$, then there exists no string of $\lambda$ and so ent $\left(\sigma_{\lambda}\right)=0$ by Theorem 4.14. If $m= \pm 1$, then $\lambda^{2}=i d_{\Gamma}$ and so by Proposition 4.4(a) $\sigma_{\lambda}^{2}=\sigma_{\lambda^{2}}=\sigma_{i d_{\Gamma}}=i d_{G_{\Gamma}}$ and by Lemma 2.5 (b) ent $\left(\sigma_{\lambda}\right)=0$.

$\left(b_{2}\right)$ Suppose that $\lambda \in \operatorname{Aut}(\Gamma)$. Then the orbits of $\lambda$ are exactly the equivalence classes of the relation $\Re_{\lambda}$ (see Example 3.3). Therefore, if $\lambda$ has infinitely many infinite orbits, $\alpha_{\lambda}$ is infinite and by Example 3.3 and Theorem $4.14 \operatorname{ent}\left(\sigma_{\lambda}\right)=+\infty$.

$\left(\mathrm{b}_{3}\right)$ Consider $\Gamma=\mathbb{Z} \times \mathbb{Z}$ and $\lambda \in \operatorname{Aut}(\Gamma)$ defined by $\lambda(x, y)=(x+y, y)$ for every $(x, y) \in \Gamma$. For every $n \in \mathbb{N}$ the orbits of $(0, n)$, that is,

$$
(0, n) / \Re_{\lambda}=\{\ldots,(-2 n, n),(-n, n),(0, n),(n, n),(2 n, n), \ldots\},
$$

are infinitely many and pairwise disjoint. Then $\operatorname{ent}\left(\sigma_{\lambda}\right)=+\infty$ by $\left(\mathrm{b}_{2}\right)$.

The next example is dedicated to the composition of generalized shifts. Let us mention that from the formulas ent $\left(\sigma_{\lambda}^{k}\right)=k \operatorname{ent}\left(\sigma_{\lambda}\right)$ (see Lemma 2.2) and $\sigma_{\lambda}^{k}=\sigma_{\lambda^{k}}$ (see Proposition 4.4), and Theorem 4.14 we obtain the useful non-obvious formula $s_{\lambda^{k}}=k s_{\lambda}$.

Example 5.6. Let $\Gamma=\mathbb{N}_{0}$ and let $\mu_{1}: \Gamma \rightarrow \Gamma$ be defined by

$$
\mu_{1}(m)= \begin{cases}p^{2 k} & \text { if } m=p^{2 k+1} \text { with } p \in \mathbb{P} \text { and } k \in \mathbb{N}, \\ p^{2 k+1} & \text { if } m=p^{2 k} \text { with } p \in \mathbb{P} \text { and } k \in \mathbb{N} \\ m & \text { otherwise. }\end{cases}
$$


Hence $\mu_{1}^{2}=i d_{\Gamma}$ and by Proposition 4.4(a) $\sigma_{\mu_{1}}^{2}=\sigma_{\mu_{1}^{2}}=\sigma_{i d_{\Gamma}}=i d_{G_{\Gamma}}$. Then ent $\left(\sigma_{\mu_{1} \circ \mu_{1}}\right)=0$ and since $\sigma_{\mu_{1}}$ is periodic, ent $\left(\sigma_{\mu_{1}}\right)=0$ by Lemma 2.5(b). The diagram for $\mu_{1}$ is the following:

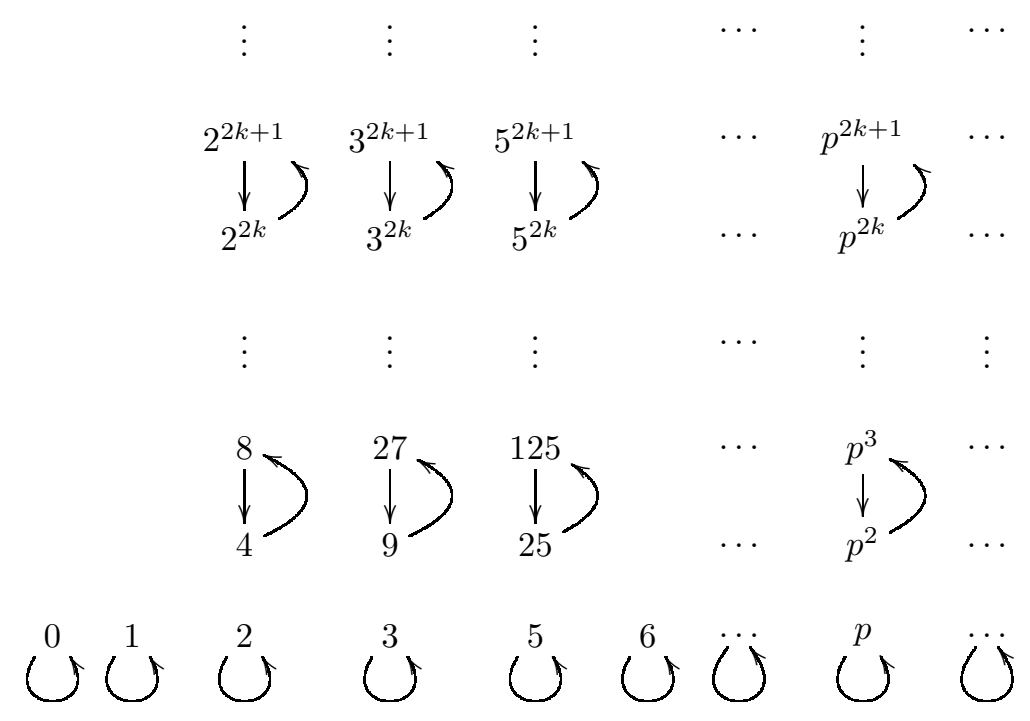

Let $\mu_{2}: \Gamma \rightarrow \Gamma$ be defined by

$$
\mu_{2}(m)= \begin{cases}p^{2 k-1} & \text { if } m=p^{2 k} \text { with } p \in \mathbb{P} \text { and } k \in \mathbb{N} \\ p^{2 k} & \text { if } m=p^{2 k-1} \text { with } p \in \mathbb{P} \text { and } k \in \mathbb{N}, \\ m & \text { otherwise (i.e., } m \text { is not a prime power) }\end{cases}
$$

Analogously, $\mu_{2}^{2}=i d_{\Gamma}$ and by Proposition 4.4(a) $\sigma_{\mu_{2}}^{2}=\sigma_{\mu_{2}^{2}}=\sigma_{i d_{\Gamma}}=i d_{G_{\Gamma}}$. Then $\operatorname{ent}\left(\sigma_{\mu_{2} \circ \mu_{2}}\right)=0$ and sice $\sigma_{\mu_{2}}$ is periodic, so ent $\left(\sigma_{\mu_{2}}\right)=0$ by Lemma 2.5(b). The diagram for $\mu_{2}$ is the following:

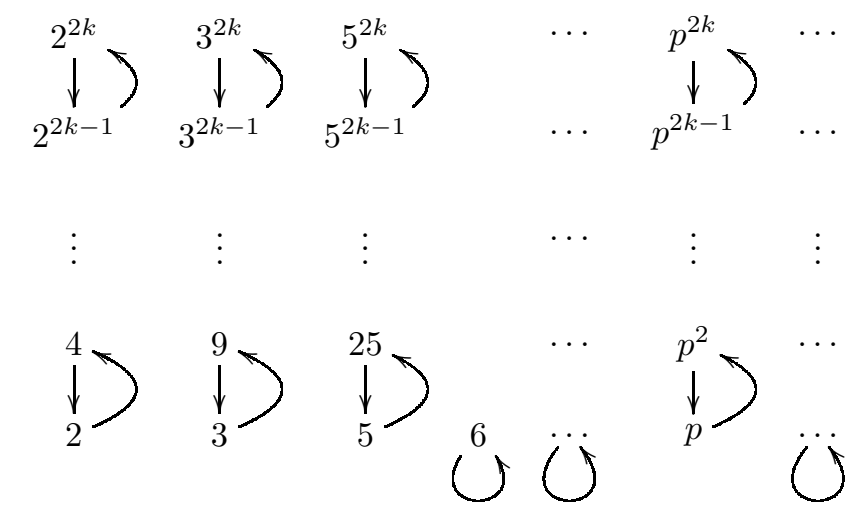


The diagram for $\mu_{1} \circ \mu_{2}: \Gamma \rightarrow \Gamma$ is the following:

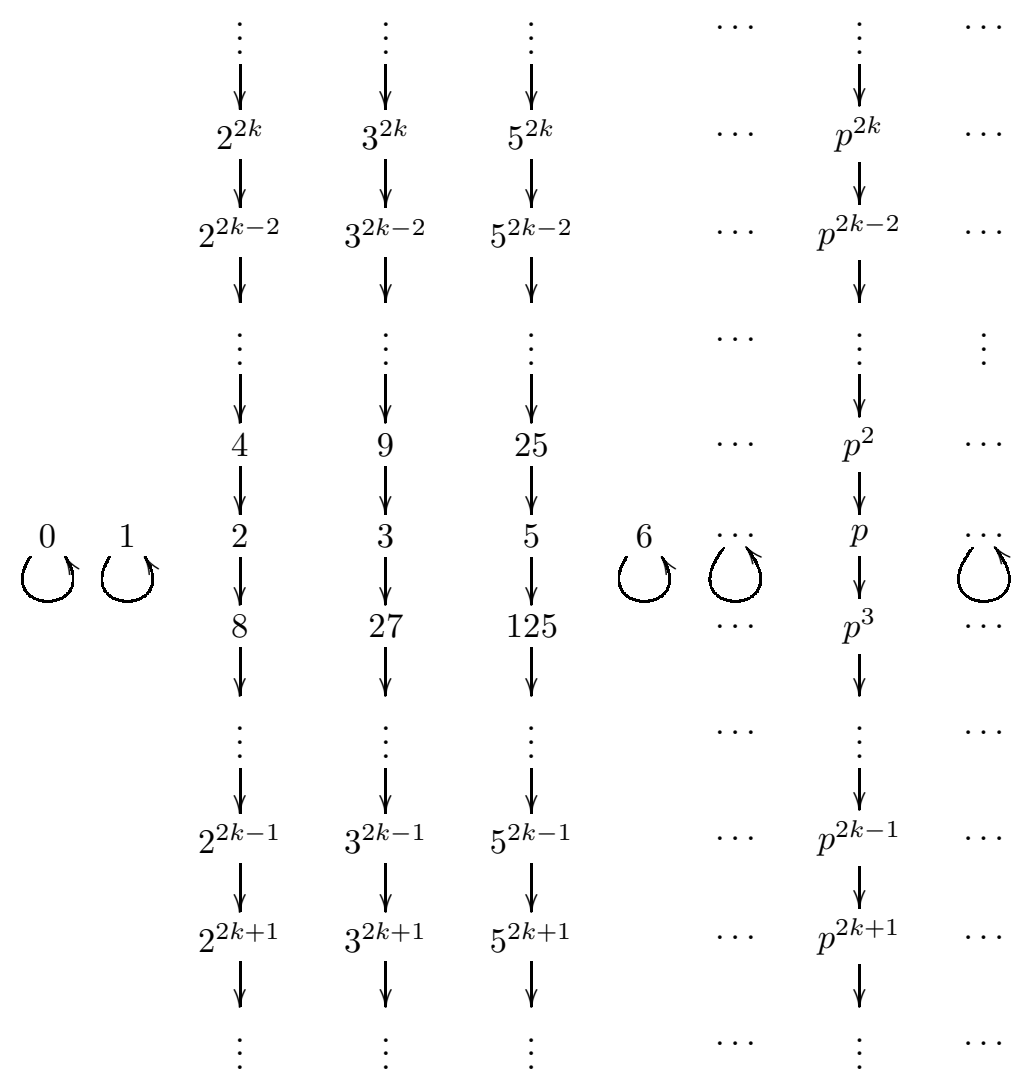

In this case $s_{\mu_{1} \circ \mu_{2}}=\alpha_{\mu_{1} \circ \mu_{2}}=\omega$ (so $\left.\left|s_{\mu_{1} \circ \mu_{2}}\right|^{*}=+\infty\right)$ and by Theorem $4.14 \operatorname{ent}\left(\sigma_{\mu_{1} \circ \mu_{2}}\right)=+\infty$. Similarly one can see that also $\mathrm{s}_{\mu_{2} \circ \mu_{1}}=\alpha_{\mu_{1} \circ \mu_{2}}=\omega$ and so that $\operatorname{ent}\left(\sigma_{\mu_{2} \circ \mu_{1}}\right)=+\infty$.

By Proposition 4.4 (a) $\sigma_{\mu_{1} \circ \mu_{2}}=\sigma_{\mu_{1}} \circ \sigma_{\mu_{2}}$; hence $\sigma_{\mu_{1}} \circ \sigma_{\mu_{2}}$ is an example of an endomorphism of infinite entropy with both $\sigma_{\mu_{1}}$ and $\sigma_{\mu_{2}}$ of entropy 0 . The same is $\sigma_{\mu_{2}} \circ \sigma_{\mu_{1}}$.

Let $\varrho_{1}: \Gamma \rightarrow \Gamma$ be defined by $\varrho_{1}(0)=1$ and $\varrho_{1}(m)=m$ for every $m \in \mathbb{N}$. Then $s_{\varrho_{1}}=0$ and so $\operatorname{ent}\left(\sigma_{\varrho_{1}}\right)=0$ by Theorem 4.14

The diagram for $\varrho_{1} \circ \varphi_{1}: \Gamma \rightarrow \Gamma$ is the following:

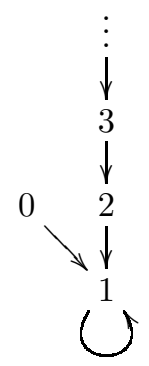

For this function $s_{\varrho_{1} \circ \varphi_{1}}=1$, and so by Theorem $4.14 \operatorname{ent}\left(\sigma_{\varrho_{1} \circ \varphi_{1}}\right)=\log |K|$.

By Proposition 4.4(a) $\sigma_{\varrho_{1} \circ \varphi_{1}}=\sigma_{\varrho_{1}} \circ \sigma_{\varphi_{1}}$; consequently $\operatorname{ent}\left(\sigma_{\varrho_{1}} \circ \sigma_{\varphi_{1}}\right)=\operatorname{ent}\left(\sigma_{\varphi_{1}}\right)=\log |K|$, while $\operatorname{ent}\left(\sigma_{\varrho_{1}}\right)=0$.

Theorem 5.7. If $\lambda: \Gamma \rightarrow \Gamma, \mu: \Upsilon \rightarrow \Upsilon$ are such that for each $(i, j) \in \Gamma \times \Upsilon, \lambda^{-1}(i)$ and $\mu^{-1}(j)$ are finite, then for the endomorphism $\sigma_{\lambda \times \mu}: G_{\Gamma \times \Upsilon} \rightarrow G_{\Gamma \times \Upsilon}$ we have:

$$
\operatorname{ent}\left(\sigma_{\lambda \times \mu}\right)=0 \text { if } \begin{cases}\operatorname{ent}\left(\sigma_{\lambda}\right)=\operatorname{ent}\left(\sigma_{\mu}\right)=0, & \left(\mathrm{a}_{0}\right) \\ \operatorname{ent}\left(\sigma_{\mu}\right)=|\operatorname{Per}(\mu)|=0 \text { and } \operatorname{ent}\left(\sigma_{\lambda}\right)>0, & \left(\mathrm{a}_{1}\right) \\ \operatorname{ent}\left(\sigma_{\lambda}\right)=|\operatorname{Per}(\lambda)|=0 \text { and } \operatorname{ent}\left(\sigma_{\mu}\right)>0 . & \left(\mathrm{a}_{2}\right)\end{cases}
$$


Moreover,

$$
\operatorname{ent}\left(\sigma_{\lambda \times \mu}\right)=\left\{\begin{array}{lll}
+\infty & \text { if } \operatorname{ent}\left(\sigma_{\lambda}\right)>0 \text { and } \operatorname{ent}\left(\sigma_{\mu}\right)>0, & \left(\mathrm{~b}_{0}\right) \\
|\operatorname{Per}(\mu)|^{*} \operatorname{ent}\left(\sigma_{\lambda}\right) & \text { if } \operatorname{ent}\left(\sigma_{\mu}\right)=0,|\operatorname{Per}(\mu)|>0 \text { and } \operatorname{ent}\left(\sigma_{\lambda}\right)>0, & \left(\mathrm{~b}_{1}\right) \\
|\operatorname{Per}(\lambda)|^{*} \operatorname{ent}\left(\sigma_{\mu}\right) & \text { if } \operatorname{ent}\left(\sigma_{\lambda}\right)=0,|\operatorname{Per}(\lambda)|>0 \text { and } \operatorname{ent}\left(\sigma_{\mu}\right)>0 . & \left(\mathrm{b}_{2}\right)
\end{array}\right.
$$

Proof. If ent $\left(\sigma_{\lambda \times \mu}\right)>0$, then by Theorem 4.14 there exists a string $\left\{\left(m_{t}, n_{t}\right)\right\}_{-t \in \mathbb{N}_{0}}$ of $\lambda \times \mu$ (for each $\left.-t \in \mathbb{N}_{0},\left(m_{t+1}, n_{t+1}\right)=\left(\lambda\left(m_{t}\right), \mu\left(n_{t}\right)\right)\right)$, therefore at least one of the sequences $\left\{m_{t}\right\}-t \in \mathbb{N}_{0}$ or $\left\{n_{t}\right\}-t \in \mathbb{N}_{0}$ is a string, which shows that either $\operatorname{ent}\left(\sigma_{\lambda}\right)>0$ or $\operatorname{ent}\left(\sigma_{\mu}\right)>0$, in view of Theorem 4.14. This proves $\left(\mathrm{a}_{0}\right)$.

Assume that $\operatorname{ent}\left(\sigma_{\mu}\right)=0$ and $\operatorname{ent}\left(\sigma_{\lambda}\right)>0$. We prove that ent $\left(\sigma_{\lambda \times \mu}\right)=0$ if $|\operatorname{Per}(\mu)|=0$. To this end, suppose that $\operatorname{ent}\left(\sigma_{\lambda \times \mu}\right)>0$. By Theorem $4.14 s_{\lambda \times \mu}>0$, so let $\left\{\left(m_{t}, n_{t}\right)\right\}_{-t \in \mathbb{N}_{0}}$ be a string of $\lambda \times \mu$. Since $\operatorname{ent}\left(\sigma_{\mu}\right)=0, s_{\mu}=0$ by Theorem 4.14, and so $\left\{m_{t}\right\}_{-t \in \mathbb{N}_{0}}$ has to be a string of $\lambda$ and $n_{0}$ is a periodic point of $\mu$. In particular $|\operatorname{Per}(\mu)|>0$. This proves $\left(\mathrm{a}_{1}\right)$.

Reverting the roles of $\lambda$ and $\mu$ one can prove $\left(\mathrm{a}_{2}\right)$.

Now let ent $\left(\sigma_{\lambda}\right)>0$ and $\operatorname{ent}\left(\sigma_{\mu}\right)>0$. By Theorem 4.14 there exist strings $\left\{m_{t}\right\}_{-t \in \mathbb{N}_{0}}$ and $\left\{n_{t}\right\}-t \in \mathbb{N}_{0}$ respectively of $\lambda$ and $\mu$. For each $-l \in \mathbb{N}_{0}$, let $z_{l}:=\left(m_{0}, n_{l}\right)$. Then $\left\{\left(m_{t}, n_{l+t}\right)\right\}_{-t \in \mathbb{N}_{0}}$ is a string of $\lambda \times \mu$ for every $l \in \mathbb{N}$, and these strings are pairwise disjoint. This means that $\left|s_{\lambda \times \mu}\right|^{*}=+\infty$ and by Theorem $4.14 \operatorname{ent}\left(\sigma_{\lambda \times \mu}\right)=+\infty$. This proves $\left(\mathrm{b}_{0}\right)$.

Assume that $\operatorname{ent}\left(\sigma_{\mu}\right)=0,|\operatorname{Per}(\mu)|>0$ and $\operatorname{ent}\left(\sigma_{\lambda}\right)>0$. We prove that

$$
\operatorname{ent}\left(\sigma_{\lambda \times \mu}\right) \leq|\operatorname{Per}(\mu)|^{*} \operatorname{ent}\left(\sigma_{\lambda}\right)
$$

If ent $\left(\sigma_{\lambda \times \mu}\right)=0$ the inequality in (5.1) is trivially satisfied. So we can assume that $\operatorname{ent}\left(\sigma_{\lambda \times \mu}\right)>0$. By Theorem 4.14 $s_{\lambda \times \mu}>0$. Let $\left\{\left(m_{t}, n_{t}\right)\right\}_{-t \in \mathbb{N}_{0}}$ be a string of $\lambda \times \mu$. Since $s_{\mu}=0$ by Theorem 4.14. $\left\{m_{t}\right\}_{-t \in \mathbb{N}_{0}}$ has to be a string of $\lambda$ and each $n_{t}$ is a periodic point of $\mu$. If $\left\{\left(m_{t}, n_{t}\right)\right\}_{-t \in \mathbb{N}_{0}}$ and $\left\{\left(m_{t}^{\prime}, n_{t}^{\prime}\right)\right\}_{-t \in \mathbb{N}_{0}}$ are disjoint strings of $\lambda \times \mu$, then either $\left\{m_{t}\right\}_{-t \in \mathbb{N}_{0}}$ and $\left\{m_{t}^{\prime}\right\}_{-t \in \mathbb{N}_{0}}$ are disjoint strings of $\lambda$ or $n_{0}$ and $n_{0}^{\prime}$ are distinct periodic points of $\mu$. This proves that $s_{\lambda \times \mu} \leq|\operatorname{Per}(\mu)| s_{\lambda}$. In particular (5.1) holds by Theorem 4.14.

We show now that under the same hypotheses, also the converse implication holds true, that is, we prove that

$$
\operatorname{ent}\left(\sigma_{\lambda \times \mu}\right) \geq|\operatorname{Per}(\mu)|^{*} \operatorname{ent}\left(\sigma_{\lambda}\right)
$$

If $\left\{m_{t}\right\}_{-t \in \mathbb{N}_{0}}$ is a string of $\lambda$, and $j \in \operatorname{Per}(\mu)$ with $\left\{j=j_{0}, j_{1}, j_{2}, \ldots, j_{s}\right\}$ the finite orbit of $j$ (i.e., $\mu\left(j_{k}\right)=j_{k+1}$ for every $k \in\{0, \ldots, s-1\}$ and $\left.\mu\left(j_{s}\right)=j\right)$, then $\left\{\left(m_{t}, j_{[t]_{s+1}}\right)\right\}-t \in \mathbb{N}_{0}$ is a string of $\lambda \times \mu$ (where, for $a \in \mathbb{Z}, b \in \mathbb{N},[a]_{b}$ denotes the remainder class of $a$ modulo $b$ ). In case $i, j$ are distinct elements of $\operatorname{Per}(\mu)$, the strings $\left\{\left(m_{t}, j_{[t]_{s+1}}\right)\right\}_{-t \in \mathbb{N}_{0}}$ and $\left\{\left(m_{t}, i_{[t]_{r+1}}\right)\right\}_{-t \in \mathbb{N}_{0}}$ (where $\left\{i=i_{0}, i_{1}, i_{2}, \ldots, i_{r}\right\}$ is the finite orbit of $i$, i.e., $\mu\left(i_{k}\right)=i_{k+1}$ for every $k \in\{0, \ldots, r-1\}$ and $\left.\mu\left(i_{r}\right)=i\right)$ are pairwise disjoint. This proves that $s_{\lambda \times \mu} \geq|\operatorname{Per}(\mu)| s_{\lambda}$, and by Theorem 4.14 (5.2) holds.

By (5.1) and (5.2) ent $\left(\sigma_{\lambda \times \mu}\right)=|\operatorname{Per}(\mu)|^{*} \operatorname{ent}\left(\sigma_{\lambda}\right)$. This concludes the proof of $\left(\mathrm{b}_{1}\right)$.

Reverting the roles of $\lambda$ and $\mu$ one can prove $\left(\mathrm{b}_{2}\right)$.

In the notations of this theorem, the following example shows that in the case where ent $\left(\sigma_{\mu}\right)=0$ and $\operatorname{ent}\left(\sigma_{\lambda}\right)=\log |K|$, it is possible that ent $\left(\sigma_{\lambda \times \mu}\right)$ is positive and also infinite, depending on the cardinality of $\operatorname{Per}(\mu)$. 
Example 5.8. Let $t \in \mathbb{N}, \Gamma_{t}=\{1, \ldots, t\}$ and $\theta_{t}=(123 \ldots t) \in S_{\Gamma_{t}}$. Let $\Lambda=\mathbb{N}_{0} \times \Gamma_{t}$. Then $\varphi_{1} \times \theta_{t}: \Lambda \rightarrow \Lambda$ and its diagram is the following:

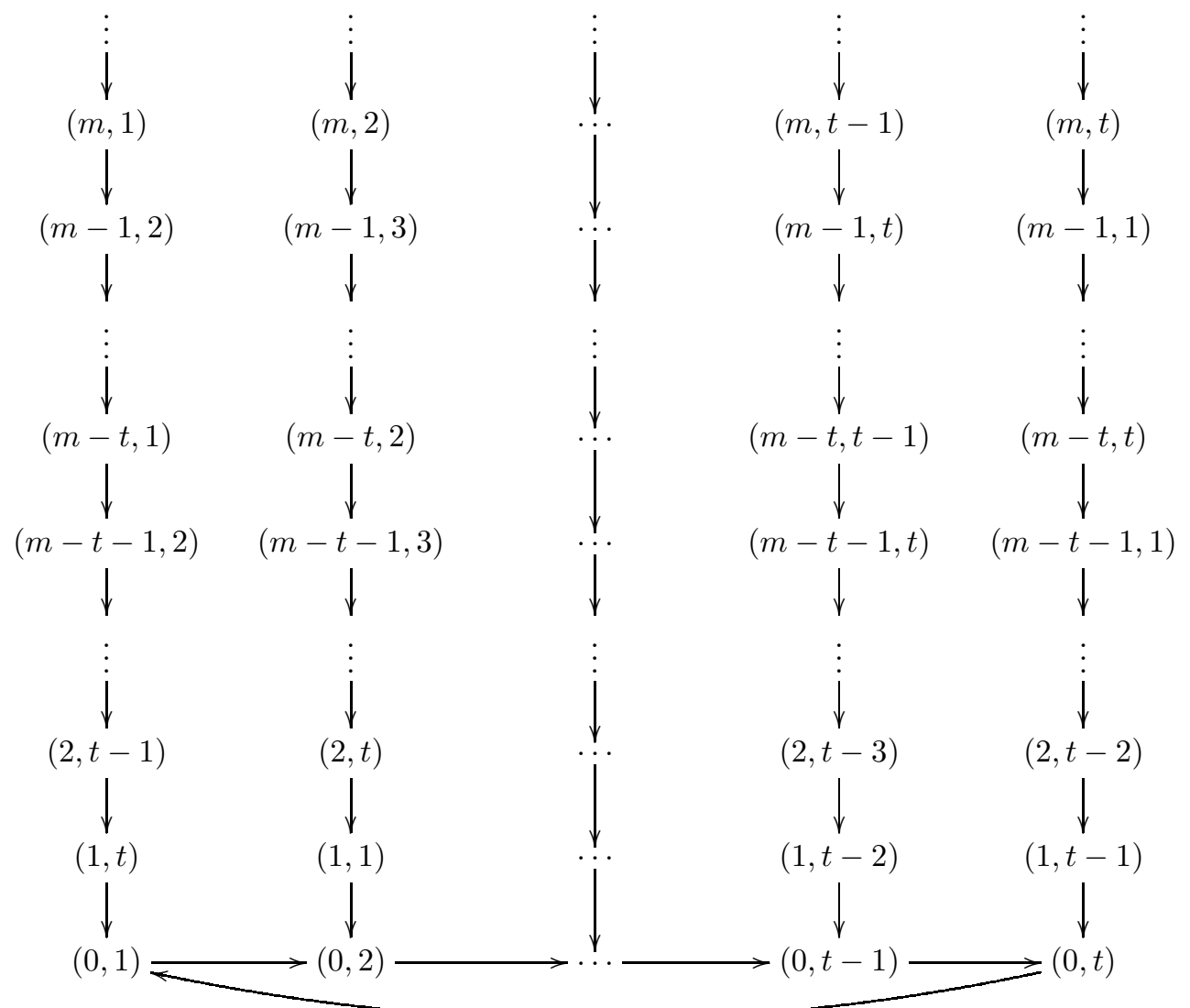

Let $\theta=(12) \in S_{\mathbb{N}}$ and let $\Lambda=\mathbb{N} \times \mathbb{N}_{0}$. The diagram for $\theta \times \varphi_{1}: \Lambda \rightarrow \Lambda$ is the following:

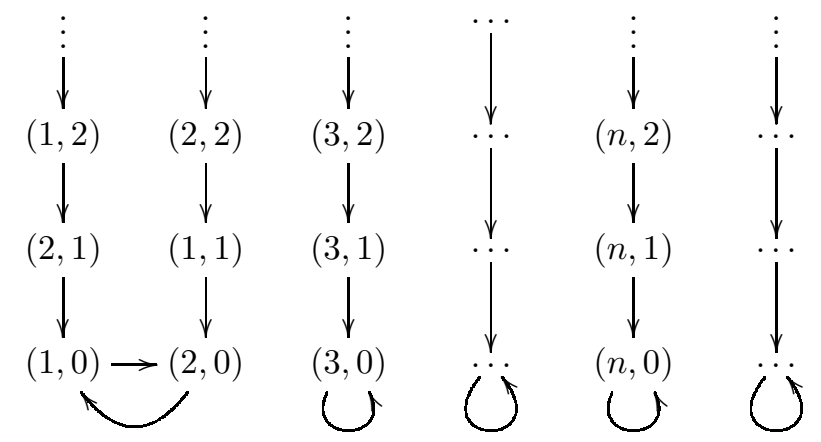

Then by Theorem $4.14 \operatorname{ent}\left(\sigma_{\varphi_{1} \times \theta_{t}}\right)=t \log |K|$ and $\operatorname{ent}\left(\sigma_{\varphi_{1} \times \theta}\right)=+\infty$, while $\operatorname{ent}\left(\sigma_{\varphi_{1}}\right)=\log |K|$ and $\operatorname{ent}\left(\sigma_{\theta}\right)=\operatorname{ent}\left(\sigma_{\theta_{t}}\right)=0$, since $\left|\operatorname{Per}\left(\theta_{t}\right)\right|=t,|\operatorname{Per}(\theta)|^{*}=+\infty$.

The next is an application of Theorem 5.7 and Corollary 5.3. Indeed considering the product of two finite abelian groups $K \times L$ instead of only one finite abelian group $K$ (as in Theorem 5.7) is not a more general situation, since Theorem 4.14, but also Corollary 5.3. shows that the entropy of a generalized shift depends mainly on its string number $s_{\lambda}$.

Corollary 5.9. Let $K$ and $L$ be finite non-trivial abelian groups and let $\lambda: \Gamma \rightarrow \Gamma$ and $\mu: \Upsilon \rightarrow \Upsilon$ be such that for each $(i, j) \in \Gamma \times \Upsilon, \lambda^{-1}(i)$ and $\mu^{-1}(j)$ are finite. Then for $\sigma_{\lambda \times \mu}:(K \times L)^{(\Gamma \times \Upsilon)} \rightarrow$ 
$(K \times L)^{(\Gamma \times \Upsilon)}$ we have:

$$
\operatorname{ent}\left(\sigma_{\lambda \times \mu}\right)=0 \text { if } \begin{cases}\operatorname{ent}\left(\sigma_{\lambda}\right)=\operatorname{ent}\left(\sigma_{\mu}\right)=0, & \left(\mathrm{a}_{0}\right) \\ \operatorname{ent}\left(\sigma_{\mu}\right)=|\operatorname{Per}(\mu)|=0 \text { and } \operatorname{ent}\left(\sigma_{\lambda}\right)>0, & \left(\mathrm{a}_{1}\right) \\ \operatorname{ent}\left(\sigma_{\lambda}\right)=|\operatorname{Per}(\lambda)|=0 \text { and } \operatorname{ent}\left(\sigma_{\mu}\right)>0 . & \left(\mathrm{a}_{2}\right)\end{cases}
$$

Moreover,

$$
\operatorname{ent}\left(\sigma_{\lambda \times \mu}\right)=\left\{\begin{array}{lll}
+\infty & \text { if } \operatorname{ent}\left(\sigma_{\lambda}\right)>0 \text { and } \operatorname{ent}\left(\sigma_{\mu}\right)>0, & \left(\mathrm{~b}_{0}\right) \\
\frac{\log |K \times L|}{\log |K|}|\operatorname{Per}(\mu)|^{*} \operatorname{ent}\left(\sigma_{\lambda}\right) & \text { if } \operatorname{ent}\left(\sigma_{\mu}\right)=0,|\operatorname{Per}(\mu)|>0 \text { and } \operatorname{ent}\left(\sigma_{\lambda}\right)>0, & \left(\mathrm{~b}_{1}\right) \\
\frac{\log |K \times L|}{\log |K|}|\operatorname{Per}(\lambda)|^{*} \operatorname{ent}\left(\sigma_{\mu}\right) & \text { if } \operatorname{ent}\left(\sigma_{\lambda}\right)=0,|\operatorname{Per}(\lambda)|>0 \text { and } \operatorname{ent}\left(\sigma_{\mu}\right)>0 . & \left(\mathrm{b}_{2}\right)
\end{array}\right.
$$

Proof. By Corollary $5.3 \operatorname{ent}\left(\sigma_{\lambda \times \mu, K \times L}\right)=\frac{\log |K \times L|}{\log |K|} \operatorname{ent}\left(\sigma_{\lambda \times \mu, K}\right)$. Now apply Theorem 5.7

In the next example we associate to a given map $\lambda$ a natural extension map $\Lambda$ such that $\operatorname{ent}\left(\sigma_{\Lambda}\right)$ is either infinite or 0 , depending on whether $\operatorname{ent}\left(\sigma_{\lambda}\right)$ is positive or 0 .

Example 5.10. Define $\Lambda: \mathcal{P}_{\text {fin }}(\Gamma) \rightarrow \mathcal{P}_{\text {fin }}(\Gamma)$ by $\Lambda(A)=\{\lambda(i): i \in A\}=\lambda(A)$ for every $A \in \mathcal{P}_{\text {fin }}(\Gamma)$. (Since $\Gamma$ embeds into $\mathcal{P}_{\text {fin }}(\Gamma)$ in a natural way via the singletons, $\Lambda$ can be considered as an extension of $\lambda$.) For each $A \in \mathcal{P}_{\text {fin }}(\Gamma), \Lambda^{-1}(A)$ is a finite subset of $\mathcal{P}_{f i n}(\Gamma)$. Consider $\sigma_{\Lambda}: G_{\mathcal{P}_{f i n}(\Gamma)} \rightarrow G_{\mathcal{P}_{f i n}(\Gamma)}$. Then:

$$
\operatorname{ent}\left(\sigma_{\Lambda}\right)= \begin{cases}0 & \text { if ent }\left(\sigma_{\lambda}\right)=0 \\ +\infty & \text { if } \operatorname{ent}\left(\sigma_{\lambda}\right)>0\end{cases}
$$

If ent $\left(\sigma_{\Lambda}\right)>0$, by Theorem 4.14 there exists at least one string $\left\{A_{t}\right\}_{-t \in \mathbb{N}_{0}}$ of $\Lambda$ in $\mathcal{P}_{\text {fin }}(\Gamma)$. In particular there exists a string $\left\{m_{t}\right\}_{-t \in \mathbb{N}_{0}}$ of $\lambda$ in $\Gamma$ : indeed, there exists $-t \in \mathbb{N}_{0}$ such that not all elements of $A_{t}$ are periodic for $\lambda$. Suppose without loss of generality that $t=0$ and let $m_{0} \in A_{0} \backslash \operatorname{Per}(\lambda)$. Then there exists an infinite sequence $\left\{m_{t}\right\}_{-t \in \mathbb{N}_{0}}$ of elements of $\Gamma$ such that $m_{t} \in A_{t}$ and $\lambda\left(m_{t}\right)=m_{t+1}$ for every $-t \in \mathbb{N}$. The elements $m_{t}$ have to be distinct because $m_{0}$ is not periodic. So $\left\{m_{t}\right\}_{-t \in \mathbb{N}_{0}}$ is a string of $\lambda$ and by Theorem $4.14 \operatorname{ent}\left(\sigma_{\lambda}\right)>0$. This proves that if $\operatorname{ent}\left(\sigma_{\lambda}\right)=0$ then $\operatorname{ent}\left(\sigma_{\Lambda}\right)=0$.

If $\operatorname{ent}\left(\sigma_{\lambda}\right)>0$, by Theorem 4.14 there exists a string $\left\{m_{t}\right\}_{-t \in \mathbb{N}_{0}}$ of $\lambda$ in $\Gamma$. For each $l \in \mathbb{N}_{0}$, $S_{l}:=\left\{\left\{m_{t}, m_{t-1}, \ldots, m_{t-l}\right\}\right\}_{-t \in \mathbb{N}_{0}}$ is a string of $\Lambda$ in $\mathcal{P}_{f i n}(\Gamma)$ and obviously $S_{1}, \ldots, S_{l}, \ldots$ are pairwise disjoint strings of $\Lambda$ in $\mathcal{P}_{\text {fin }}(\Gamma)$. So Theorem 4.14 leads us to the desired result, that is $\operatorname{ent}\left(\sigma_{\Lambda}\right)=+\infty$.

\section{FinAl REMARKS AND OPEN PROBLEMS}

We consider here the generalized shift $\sigma_{\lambda}$ on $K^{\Gamma}$ and, in case $\lambda$ has finite fibers, its restriction $\sigma_{\lambda}^{\oplus}$ on $K^{(\Gamma)}$. Theorem 4.14 calculates precisely the value of the entropy of $\sigma_{\lambda}^{\oplus}: K^{(\Gamma)} \rightarrow K^{(\Gamma)}$. The necessary property on $\lambda$ to have finite fibers helps us in finding the explicit formula for the entropy of $\sigma_{\lambda}^{\oplus}$. In the general case of $\sigma_{\lambda}: K^{\Gamma} \rightarrow K^{\Gamma}$ we leave open the following problem.

Problem 6.1. Calculate the entropy of $\sigma_{\lambda}: K^{\Gamma} \rightarrow K^{\Gamma}$. Is $\operatorname{ent}\left(\sigma_{\lambda}\right)=\operatorname{ent}\left(\sigma_{\lambda}^{\oplus}\right)$ in case $\lambda$ has finite fibers?

Note that $\operatorname{ent}\left(\sigma_{\lambda}\right) \geq \operatorname{ent}\left(\sigma_{\lambda}^{\oplus}\right)$ in the latter case, since then $K^{(\Gamma)}$ is $\sigma_{\lambda}$-invariant in $K^{\Gamma}$.

Problem 6.2. $\quad$ (a) Is it possible to have $\operatorname{ent}\left(\sigma_{\lambda}^{\oplus}\right)=0$, but $\operatorname{ent}\left(\sigma_{\lambda}\right)>0$ ?

(b) Is it possible to have $0<\operatorname{ent}\left(\sigma_{\lambda}\right)<+\infty$ ?

Moreover, Theorem 4.14 concerns only a single abelian group $K$. If $\left\{K_{i}: i \in \Gamma\right\}$ is a family of abelian groups and $\rho_{i}: K_{\lambda(i)} \rightarrow K_{i}$ is a homomorphism for every $i \in I$, define $\widetilde{\sigma}_{\lambda}: \prod_{i \in \Gamma} K_{i} \rightarrow \prod_{i \in \Gamma} K_{i}$ by $\widetilde{\sigma}_{\lambda}(x)=\left(\rho_{i}\left(x_{\lambda(i)}\right)\right)_{i \in \Gamma}$ for every $x=\left(x_{i}\right)_{i \in \Gamma} \in \prod_{i \in \Gamma} K_{i}$. It is possible to consider also $\widetilde{\sigma}_{\lambda}$ restricted to the direct sum, that is, $\tilde{\sigma}_{\lambda}^{\oplus}: \bigoplus_{i \in \Gamma} K_{i} \rightarrow \bigoplus_{i \in \Gamma} K_{i}$.

Problem 6.3. Suppose that for every $i \in \Gamma K_{i}$ is a finite abelian group.

(a) Calculate the entropy of $\widetilde{\sigma}_{\lambda}: \prod_{i \in \Gamma} K_{i} \rightarrow \prod_{i \in \Gamma} K_{i}$.

(b) Calculate the entropy of $\tilde{\sigma}_{\lambda}^{\oplus}: \bigoplus_{i \in \Gamma} K_{i} \rightarrow \bigoplus_{i \in \Gamma} K_{i}$. 
A particular case of this problem is when for each $i \in \Gamma K_{\lambda(i)} \leq K_{i}$, that is, $\rho_{i}: K_{\lambda(i)} \rightarrow K_{i}$ is an injective homomorphism. So one can consider first the problem in this case.

Let $K$ be a finite field and $R=K[x]$. For $r \in R$, let $m_{r}: R \rightarrow R$ be defined by $m_{r}(s)=r s$ for every $s \in R$. It is easy to see that for the natural isomorphism $j: K[x] \rightarrow K^{\left(\mathbb{N}_{0}\right)}$ the conjugated isomorphism $j \circ m_{x} \circ j^{-1}$ coincides with the right Bernoulli shift $\beta_{K}$ of $K^{\left(\mathbb{N}_{0}\right)}$ (and consequently, $j \circ m_{x^{n}} \circ j^{-1}=\beta_{K}^{n}$ ). Therefore, the endomorphism $m_{r}$ is (conjugated to) a linear combination of powers of the Bernoulli shift $\beta_{K}$.

Problem 6.4. Calculate the entropy of $m_{r}: K[x] \rightarrow K[x]$. What about the ring $K\left[x_{1}, \ldots, x_{n}\right]$ of polynomials in more variables?

This problem can be generalized for graded rings. (Let us recall that a graded ring is a ring $R$ with a family $\left\{R_{i}: i \in \mathbb{N}_{0}\right\}$ of subgroups of $(R,+)$ such that $R=\bigoplus_{i=0}^{\infty} R_{i}$ and $R_{i} R_{j} \subseteq R_{i+j}$ for all $i, j \in \mathbb{N}_{0}$ [2, Chapter 10].) For $r \in R$, let $m_{r}: R \rightarrow R$ be defined by $m_{r}(s)=r s$ for every $s \in R$.

Problem 6.5. Compute the entropy ent $\left(m_{r}\right)$ in case $R$ is a graded ring, $r \in R$ and $m_{r}: R \rightarrow R$.

Problem 6.5 can be extended also to graded $R$-modules $M$ and the endomorphism $m_{r}$ of $M$ defined by the multiplication, in $M$, by a fixed element $r \in R$ as above.

We conclude with a problem suggested by Example 5.5(b).

Problem 6.6. Let $\Gamma$ be an abelian group and $\lambda: \Gamma \rightarrow \Gamma$ an endomorphism.

(a) Is it true that $s_{\lambda}>0$ implies $s_{\lambda}$ infinite?

(b) Describe in which cases $s_{\lambda}=0$ and in which cases $s_{\lambda}$ is infinite.

\section{REFERENCES}

[1] R. L. Adler, A. G. Konheim, M. H. McAndrew, Topological entropy, Trans. Amer. Math. Soc. 114 (1965), $309-319$.

[2] M. F. Atiyah, I. G. MacDonald, Introduction to commutative algebra, Addison-Wesley Publishing Co., 1969.

[3] F. Ayatollah Zadeh Shirazi, N. Karami Kabir, F. Heidari Ardi, A note on shift theory, Math. Pannon. 19 (2008) no. 2, 187-195.

[4] D. Dikranjan, B. Goldsmith, L. Salce, P. Zanardo, Algebraic entropy for abelian groups, Trans. Amer. Math. Soc. 361 (2009), 3401-3434.

[5] J. König, Zum Kontinuumproblem, Mathematische Annalen 60 (1904), 177-180.

[6] M. D. Weiss, Algebraic and other entropies of group endomorphisms, Math. Systems Theory 8 (1974/75) no. 3, $243-248$.

(Maryam Akhavin) Faculty of Mathematical Sciences, University for Teacher Education, 599 Taleghani Ave., Tehran 15614, Iran

E-mail address: m_akhavin@tmu.ac.ir

(Fatemah Ayatalloh Zadeh Shirazi) Faculty of Mathematics, Statistics and Computer Science, College of Science, University of Tehran, Enghelab Ave., Tehran, Iran

E-mail address: fatemah@khayam.ut.ac.ir

(Dikran Dikranjan) Università di Udine, Dipartimento di Matematica e Informatica, via delle Scienze, 206 33100 Udine, ItAly

E-mail address: dikran.dikranjan@dimi.uniud.it

(Anna Giordano Bruno) Università di Udine, Dipartimento di Matematica e Informatica, via delle Scienze, 206 - 33100 Udine, Italy

E-mail address: anna.giordanobruno@dimi.uniud.it

(Arezoo Hosseini) Department of Mathematics, Faculty of Science, University of Guilan, Manzarieh Ave., RASHT, IRAN

E-mail address: a_hosseini@guilan.ac.ir 